\title{
A Stochastic Wavelet Finite Element Method for 1D and 2D Structures Analysis
}

\author{
Xingwu Zhang, Xuefeng Chen, Zhibo Yang, Bing Li, and Zhengjia He \\ The State Key Laboratory for Manufacturing Systems Engineering, School of Mechanical Engineering, \\ Xian Jiaotong University, Xian 710049, China \\ Correspondence should be addressed to Xuefeng Chen; chenxf@mail.xjtu.edu.cn
}

Received 12 November 2013; Revised 8 April 2014; Accepted 22 April 2014; Published 8 May 2014

Academic Editor: Reza Jazar

Copyright (c) 2014 Xingwu Zhang et al. This is an open access article distributed under the Creative Commons Attribution License, which permits unrestricted use, distribution, and reproduction in any medium, provided the original work is properly cited.

A stochastic finite element method based on B-spline wavelet on the interval (BSWI-SFEM) is presented for static analysis of 1D and $2 \mathrm{D}$ structures in this paper. Instead of conventional polynomial interpolation, the scaling functions of BSWI are employed to construct the displacement field. By means of virtual work principle and BSWI, the wavelet finite elements of beam, plate, and plane rigid frame are obtained. Combining the Monte Carlo method and the constructed BSWI elements together, the BSWI-SFEM is formulated. The constructed BSWI-SFEM can deal with the problems of structural response uncertainty caused by the variability of the material properties, static load amplitudes, and so on. Taking the widely used Timoshenko beam, the Mindlin plate, and the plane rigid frame as examples, numerical results have demonstrated that the proposed method can give a higher accuracy and a better constringency than the conventional stochastic finite element methods.

\section{Introduction}

The finite element method (FEM) is well accepted now in numerous industrial areas [1-4]. However, the validity of the results obtained by FEM can be drastically limited by the uncertainties of the parameters introduced in the model. Many factors may cause the uncertainties, such as a consequence of ignorance and spatial variability $[5,6]$.

On the other hand, the reliability design and analysis for engineering structures also require a kind of FE model which could deal with the variability and uncertainty. For example, a slight change in design variables may result in a poignant change of structural properties. As a powerful tool in computational stochastic mechanics, the stochastic finite element method (SFEM) appeared.

SFEM is an extension of the classical FEM to stochastic framework. The most important factor of SFEM is the uncertainty modeling. Over the past several decades, many methods were combined with FEM to approximate the uncertainties mentioned above. Chakraborty and Dey investigated the stochastic finite element simulation based on the Neumann expansion of uncertain structures subjected to earthquake [7]. A method named first-order, second-moment (FOSM) method was proposed by Cornell [8]; in fact it is a special case of perturbation method $[9,10]$. Valdebenito et al. studied the application of first-order expansion considering intervening variables for estimating second-order statistics for stochastic finite element analysis [11]. The KarhunenLoeve [12] expansion, which can be seen as a kind of the orthogonal series expansion, is also an important representation of SFEM fields. Sakamoto and Ghanem [13] proposed a method based on polynomial chaos expansion for presenting the variability. Proppe implemented multiresolution analysis for stochastic finite element problems with Karhunen-Loeve expansion [14]. Based on Karhunen-Loeve series expansion and hierarchical approach, Yang et al. applied the hierarchical stochastic finite element method for structural analysis [15]. Another method is Monte Carlo simulation (MCS) [16]; the name "Monte Carlo" was coined in the 1940s by scientists working in Los Alamos to designate a class of numerical methods based on the use of random numbers. Compared with other technologies, MCS is more direct and widely used. Taking a certain amount of samples as the priori testing data, MCS can give an accurate approximation for the stochastic field [17]. Besides, Diazdelao and Adhikari explored a method to reduce the computation cost of stochastic finite element 
codes based on the Gaussian process emulation [18]. Han and Bang performed the probabilistic optimal safety assessment of steel cable-stayed bridges using SFEM and expected life cycle cost concept [19]. Lang et al. investigated the prediction of heat transfer in composite materials with uncertain geometry based on the combination of the extended finite element method and the spectral stochastic finite element method [20]. Augustin and Rentrop constructed the stochastic Galerkin techniques for the random ordinary differential equations and demonstrated the advantages through numerical examples [21]. Shang and Yun proposed a stochastic finite element within a general purpose finite element analysis program ABAQUS to simulate the probabilistic structural response of stochastic materials [22].

Another important factor of SFEM is the properties of FEM element which influence the presentation directly. Monte Carlo simulation is widely used to solve complex mechanics and physics problems, particularly those involving multiple independent stochastic variables. The essential characteristic of Monte Carlo method is the use of random numbers and random variables. A random variable is a quantity that results from a repeatable process and whose actual values cannot be predicted with certainty [23]. The occurrence frequency of a sample value would be deemed to be the approximation to its probability. With the famous "law of large number," the correctness of Monte Carlo simulation can be understood easily. Usually, a sample size $N$ of the order 30-100 could already provide estimates with acceptable accuracy [24]. On the other hand, the law makes the exactness of Monte Carlo simulation heavily depend on the sampling counter. With the growing number of sampling counters, the calculation efficiency seems to be particularly important. That is to say, the realization of MCS should be based not only on an effective computer system but also on an efficient algorithm. Therefore, a kind of new stochastic finite element method based on B-spline wavelet on the interval and MCS (BSWI-SFEM) is constructed to deal with the current problems in SFEM analysis.

It is necessary to recommend wavelet analysis and BSWI briefly before we introduce BSWI-FEM. Wavelet analysis is a new method developed in recent years [25]. The wavelet method can be viewed as a method in which the approximating function is defined by a multiresolution technique based on scaling or wavelet functions, similar to those used in signal and image processing. With its desirable characteristics, such as multiresolution properties and various basis functions for structural analysis, wavelet method is well argued by many researchers not only in numerical analysis domains [26-28] but also in structural analysis fields [29-31]. As a kind of wavelet, BSWI basis has the good characteristics of compact support, smoothness, and symmetry besides the multiresolution analysis. Moreover, it has the explicit expression, which will not lead to any trouble for differentiation and integration. So it is the best one among all existing wavelets in approximation of numerical calculation [32]. Based on the good characteristics of BSWI, several investigators have tried to combine it with FEM to solve practical problems [33-37]. However, the above-mentioned methods, which are all constructed in a determined wavelet space, can hardly make a satisfying approximation for the influence caused by structural uncertainties.

In this paper, a new BSWI-SFEM is presented. Combining BSWI element with Monte Carlo method, a BSWI-SFEM formulation is constructed to deal with the static and vibration analysis of $1 \mathrm{D}$ and $2 \mathrm{D}$ structures. Taking the Timoshenko beam, the Mindlin plate, and the plane rigid frame as examples, numerical results indicate that the proposed method has a higher accuracy and a better constringency than the conventional SFEM.

\section{The Construction of BSWI Element}

2.1. One-Dimensional BSWI on the Interval $[0,1]$. Gos wami et al. constructed the scaling functions of BSWI and gave the $m$ th order wavelet formulations at scale $j=0$ in 1995 [39]. To any scale $j$, the $m$ th order BSWI scaling functions must satisfy the following condition [39] in order to have at least one inner wavelet on the interval $[0,1]$ :

$$
2^{j} \geq 2 m-1
$$

Then $j$ scale $m$ th order BSWI (BSWI $m_{j}$ ) scaling functions $\phi_{m, k}^{j}(\xi)$ and the corresponding wavelets $\psi_{m, k}^{j}(\xi)$ can be evaluated by the following formulas:

$$
\begin{aligned}
& \phi_{m, k}^{j}(\xi)= \begin{cases}\phi_{m, k}^{l}\left(2^{j-l} \xi\right), \quad k=-m+1, \ldots,-1 & (0 \text { boundary scaling functions }) \\
\phi_{m, 2^{j}-m-k}^{l}\left(1-2^{j-l} \xi\right), \quad k=2^{j}-m+1, \ldots, 2^{j}-1 & (1 \text { boundary scaling functions }) \\
\phi_{m, 0}^{l}\left(2^{j-l} \xi-2^{-l} k\right), \quad k=0, \ldots, 2^{j}-m & \text { (inner scaling functions) }\end{cases} \\
& \psi_{m, k}^{j}(\xi)= \begin{cases}\psi_{m, k}^{l}\left(2^{j-l} \xi\right), \quad k=-m+1, \ldots,-1 & (0 \text { boundary wavelet functions) } \\
\psi_{m, 2^{j}-m-k}^{l}\left(1-2^{j-l} \xi\right), \quad k=2^{j}-m+1, \ldots, 2^{j}-1 & (1 \text { boundary wavelet functions) } \\
\psi_{m, 0}^{l}\left(2^{j-l} \xi-2^{-l} k\right), \quad k=0, \ldots, 2^{j}-m & \text { (inner wavelet functions). }\end{cases}
\end{aligned}
$$

Let $j_{0}$ be the scale which satisfies (1); then let $l=0$ in (2), for any $j>j_{0}$; according to the 0 scale $m$ th order scaling functions and wavelets given in [39], we can get the $j$ scale $m$ th order scaling functions and wavelets. There are $m-1$ 
boundary scaling functions and wavelets, $2^{j}-m+1$ inner scaling functions, and $2^{j}-2 m+2$ inner wavelets. The scaling functions and the wavelets on the interval $[0,1]$ can be written in vector form as follows:

$$
\begin{aligned}
& \boldsymbol{\Phi}=\left\{\begin{array}{lllll}
\phi_{m,-m+1}^{j}(\xi) & \phi_{m,-m+2}^{j}(\xi) & \cdots & \phi_{m, 2^{j}-1}^{j}(\xi)
\end{array}\right\}, \\
& \Psi=\left\{\begin{array}{lllll}
\psi_{m,-m+1}^{j}(\xi) & \psi_{m,-m+2}^{j}(\xi) & \cdots & \psi_{m, 2^{j}-1}^{j}(\xi)
\end{array}\right\} .
\end{aligned}
$$

The corresponding eleven scaling functions and eleven wavelets of $\mathrm{BSWI}_{3}$ are given in Figure 1.

2.2. Two-Dimensional Tensor Product BSWI on the Interval $[0,1]$. By means of tensor product, 2D BSWI can be easily extended from 1D BSWI. A separable wavelet semiorthonormal basis at scale $j$ of $\mathbf{L}^{2}\left(\mathbf{R}^{2}\right)$ is constructed with tensor products of the multiresolution approximation spaces $\mathbf{V}_{j}^{1}$ and $\mathbf{V}_{j}^{2}$, so the tensor product subspace can be expressed as $\mathbf{F}_{j}=$ $\mathbf{V}_{j}^{1} \otimes \mathbf{V}_{j}^{1}$, and the scaling functions can be expressed as

$$
\Phi=\Phi_{1} \otimes \Phi_{2}
$$

where $\boldsymbol{\Phi}_{1}=\left\{\phi_{m,-m+1}^{j}(\xi) \phi_{m,-m+2}^{j}(\xi) \cdots \phi_{m, 2^{j}-1}^{j}(\xi)\right\}$, indicating one row vector combined by the $m$ th order scaling functions at scale $j . \quad \Phi_{2}=$ $\left\{\phi_{m,-m+1}^{j}(\eta) \phi_{m,-m+2}^{j}(\eta) \cdots \phi_{m, 2^{j}-1}^{j}(\eta)\right\}$, indicating another row vector combined by the $m$ th order scaling functions at scale $j$. The wavelets functions are

$$
\begin{aligned}
& \Psi^{1}=\Phi_{1} \otimes \psi_{2} \\
& \Psi^{2}=\psi_{1} \otimes \Phi_{2} \\
& \Psi^{3}=\psi_{1} \otimes \psi_{2},
\end{aligned}
$$

where $\psi_{1}$ and $\psi_{2}$ are wavelets of BSWI $m_{j}$ in (4). Figure 2(a) shows all the scaling functions of 2D tensor products BSWI, and the wavelets of tensor products BSWI are shown in Figures 2(b), 2(c), and 2(d).

2.3. The BSWI Timoshenko Beam Element. Considering the influence of shear deformation, the generalized function of potential energy of the Timoshenko beam element can be given as [32]

$$
\begin{aligned}
\Pi_{p}(w, \theta)= & \int_{0}^{L} \frac{E I}{2}\left(-\frac{d \theta}{d x}\right)^{2} d x+\int_{0}^{L} \frac{G A}{2 k}\left(\frac{d w}{d x}-\theta\right)^{2} d x \\
& -\int_{0}^{L} f(x) w d x-\sum_{i} P_{i} w\left(x_{i}\right)+\sum_{i} M_{i} \theta\left(x_{i}\right),
\end{aligned}
$$

where $E I$ is the bending rigidity, $\theta$ is cross section rotation, $G$ is shear modulus, $A$ is the area of cross section, $P_{i}$ is the lump force, $M_{i}$ is the lump bending moment, and $k$ is the shear deformation coefficient. According to the Timoshenko beam theory, $w(\xi)$ and $\theta(\xi)$ can be independently interpolated by BSWI scaling functions as

$$
w(\xi)=\boldsymbol{\Phi} \mathbf{T}^{e} \mathbf{w}^{e}, \quad \theta(\xi)=\boldsymbol{\Phi} \mathbf{T}^{e} \boldsymbol{\theta}^{e}
$$

Translating (7) into interval $[0,1]$ and taking (8) into (7), let $\delta \Pi_{p}=0$, and the element solving equation of bending problem can be obtained as follows:

$$
\left[\begin{array}{ll}
\mathbf{K}^{e, 1} & \mathbf{K}^{e, 2} \\
\mathbf{K}^{e, 3} & \mathbf{K}^{e, 4}
\end{array}\right]\left[\begin{array}{l}
\mathbf{w}^{e} \\
\boldsymbol{\theta}^{e}
\end{array}\right]=\left[\begin{array}{c}
\mathbf{P}_{w}^{e} \\
0
\end{array}\right]+\left[\begin{array}{c}
\mathbf{P}_{w_{i}}^{e} \\
\mathbf{M}_{\theta_{l}}^{e}
\end{array}\right]
$$

where

$$
\begin{gathered}
\mathbf{P}_{w}^{e}=l_{e} \int_{0}^{1}\left(\mathbf{T}^{e}\right)^{\mathrm{T}} \boldsymbol{\Phi}^{\mathrm{T}} f(\xi) d \xi, \quad \mathbf{P}_{w_{i}}^{e}=\sum_{i} P_{i}\left(\mathbf{T}^{e}\right)^{\mathrm{T}} \boldsymbol{\Phi}^{\mathrm{T}}\left(\xi_{i}\right), \\
\mathbf{M}_{\theta_{l}}^{e}=-\sum_{l} M_{l}\left(\mathbf{T}^{e}\right)^{\mathrm{T}} \boldsymbol{\Phi}^{\mathrm{T}}\left(\xi_{l}\right) ; \\
\mathbf{K}^{e, 1}=\frac{G A}{k l_{e}} \int_{0}^{1}\left(\mathbf{T}^{e}\right)^{\mathrm{T}}\left(\frac{d \boldsymbol{\Phi}^{\mathrm{T}}}{d \xi}\right)\left(\frac{d \boldsymbol{\Phi}}{d \xi}\right) \mathbf{T}^{e} d \xi, \\
\mathbf{K}^{e, 2}=-\frac{G A}{k} \int_{0}^{1}\left(\mathbf{T}^{e}\right)^{\mathrm{T}}\left(\frac{d \boldsymbol{\Phi}^{\mathrm{T}}}{d \xi}\right) \mathbf{\Phi} \mathbf{T}^{e} d \xi, \\
\mathbf{K}^{e, 4}=\frac{E I}{l_{e}} \int_{0}^{1}\left(\mathbf{T}^{e}\right)^{\mathrm{T}}\left(\frac{d \boldsymbol{\Phi}^{\mathrm{T}}}{d \xi}\right)\left(\frac{d \boldsymbol{\Phi}}{d \xi}\right) \mathbf{T}^{e} d \xi \\
+\frac{G A l_{e}}{k} \int_{0}^{1}\left(\mathbf{T}^{e}\right)^{\mathrm{T}} \boldsymbol{\Phi}^{\mathrm{T}} \boldsymbol{\Phi} \mathbf{T}^{e} d \xi .
\end{gathered}
$$

The element solving equation of vibration problem is

$$
|\mathbf{K}-\lambda \mathbf{M}|=0,
$$

where $\mathbf{M}$ is the consistent mass matrix as follows:

$$
\mathbf{M}=\rho A l_{e}\left(\mathbf{T}^{e}\right)^{\mathrm{T}}\left(\frac{d \boldsymbol{\Phi}^{\mathrm{T}}}{d \xi}\right)\left(\frac{d \boldsymbol{\Phi}}{d \xi}\right) \mathbf{T}^{e}
$$

$\mathbf{K}$ is the stiffness matrix as follows:

$$
\mathbf{K}=\left[\begin{array}{ll}
\mathbf{K}^{e, 1} & \mathbf{K}^{e, 2} \\
\mathbf{K}^{e, 3} & \mathbf{K}^{e, 4}
\end{array}\right]
$$

$\rho$ is density and $\lambda$ is the vibration engine value.

2.4. The BSWI Mindlin Plate Element. According to the Mindlin plate theory, the displacements and rotations are 


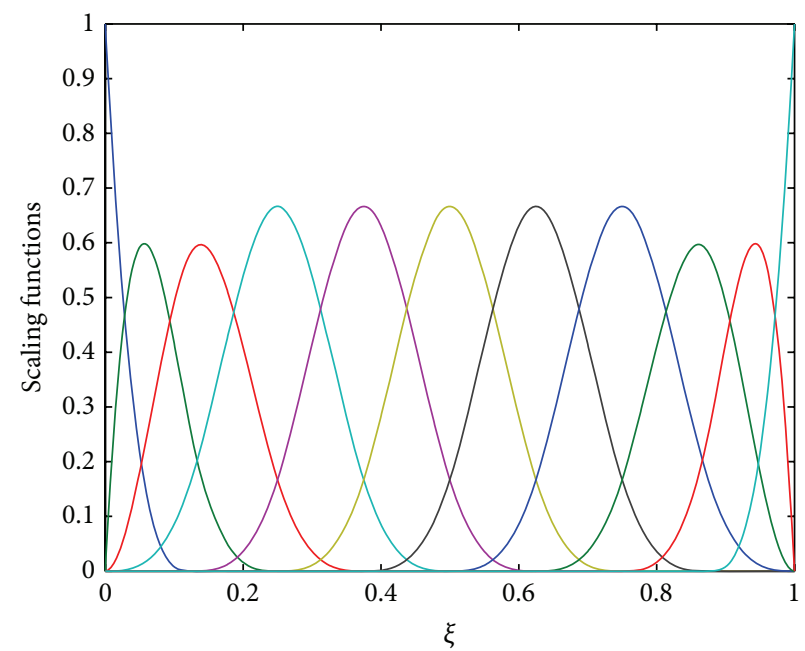

(a)

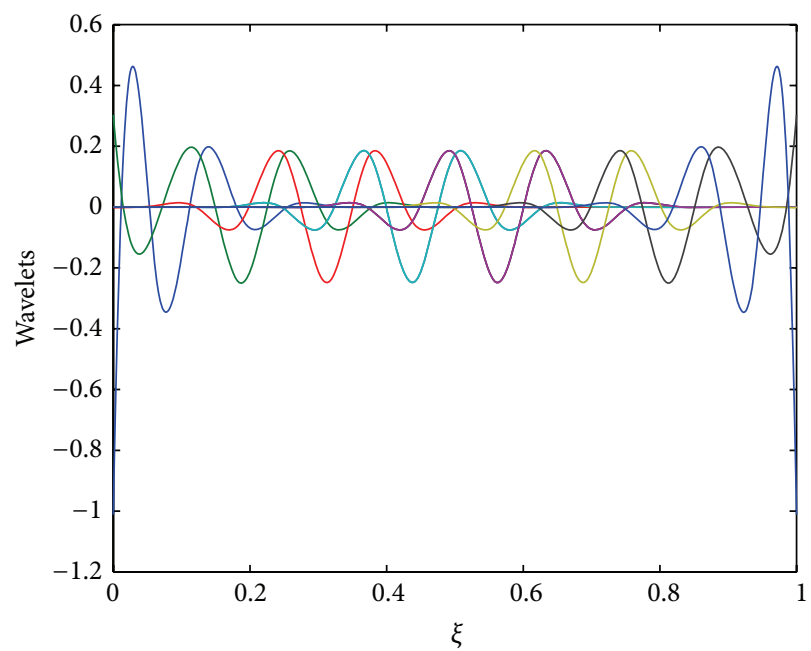

(b)

FIGURE 1: BSWI4 $4_{3}$ on the interval [0,1]: (a) scaling functions and (b) wavelets.

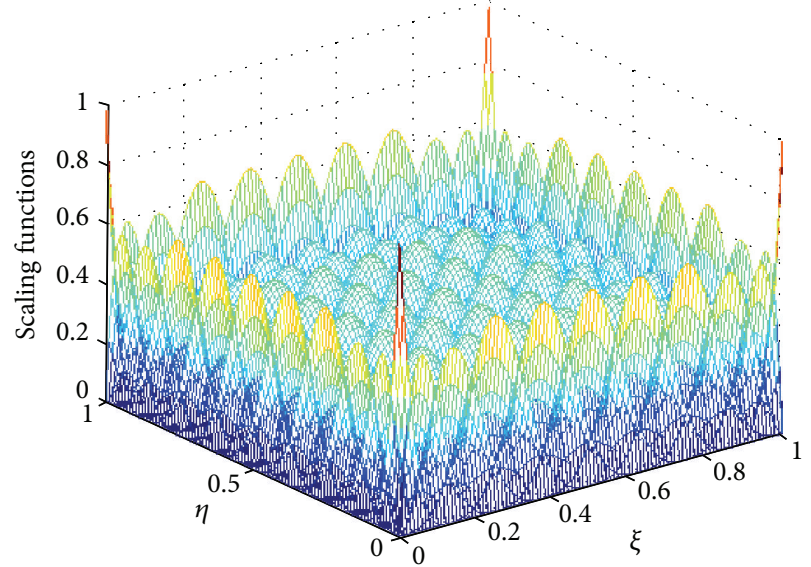

(a)

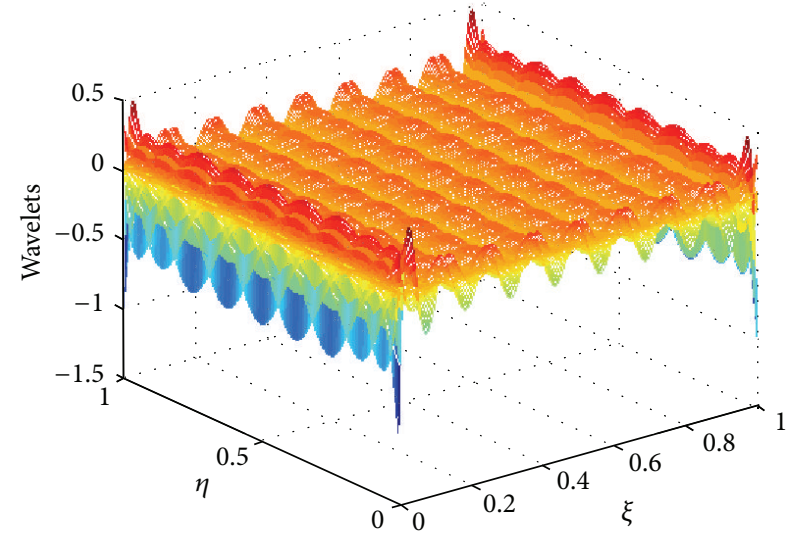

(c)

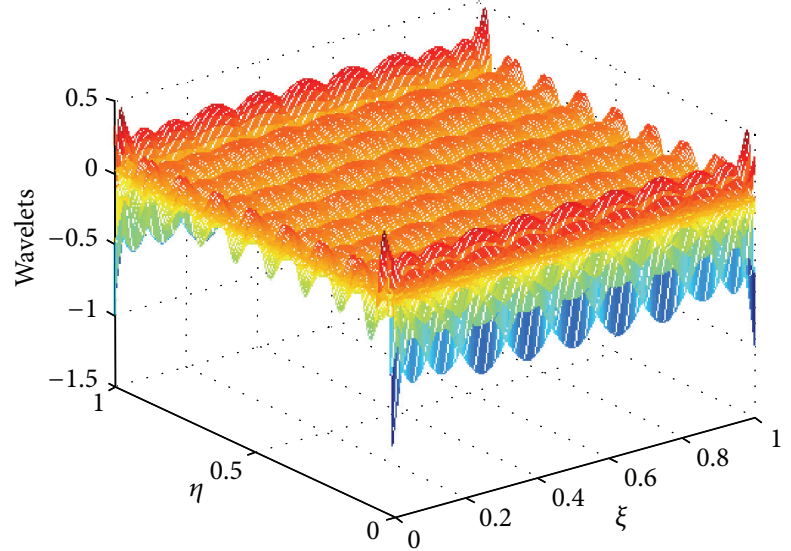

(b)

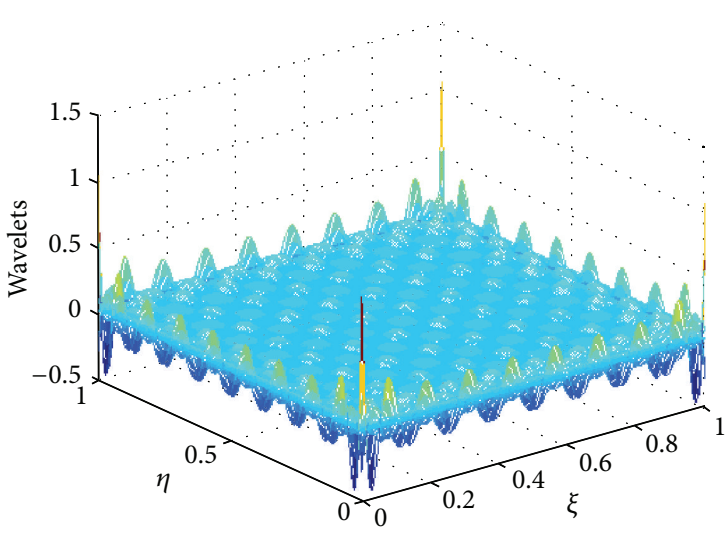

(d)

Figure 2: Two-dimensional tensor product BSWI: (a) scaling functions $\boldsymbol{\Phi}=\boldsymbol{\Phi}_{1} \otimes \boldsymbol{\Phi}_{2}$, (b) wavelets $\boldsymbol{\Psi}^{1}=\boldsymbol{\Phi}_{1} \otimes \boldsymbol{\psi}_{2}$, (c) wavelets $\boldsymbol{\Psi}^{2}=\boldsymbol{\psi}_{1} \otimes \boldsymbol{\Phi}_{2}$, and (d) wavelets $\Psi^{3}=\psi_{1} \otimes \psi_{2}$. 


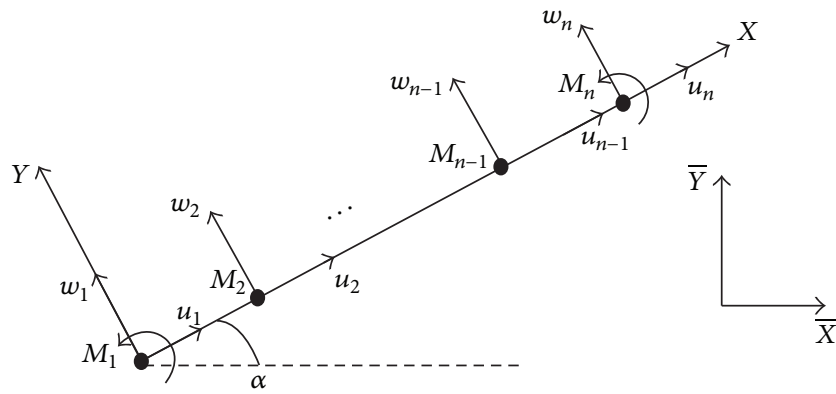

FIGURE 3: The solving domain and nodes displacement of plane rigid frame.

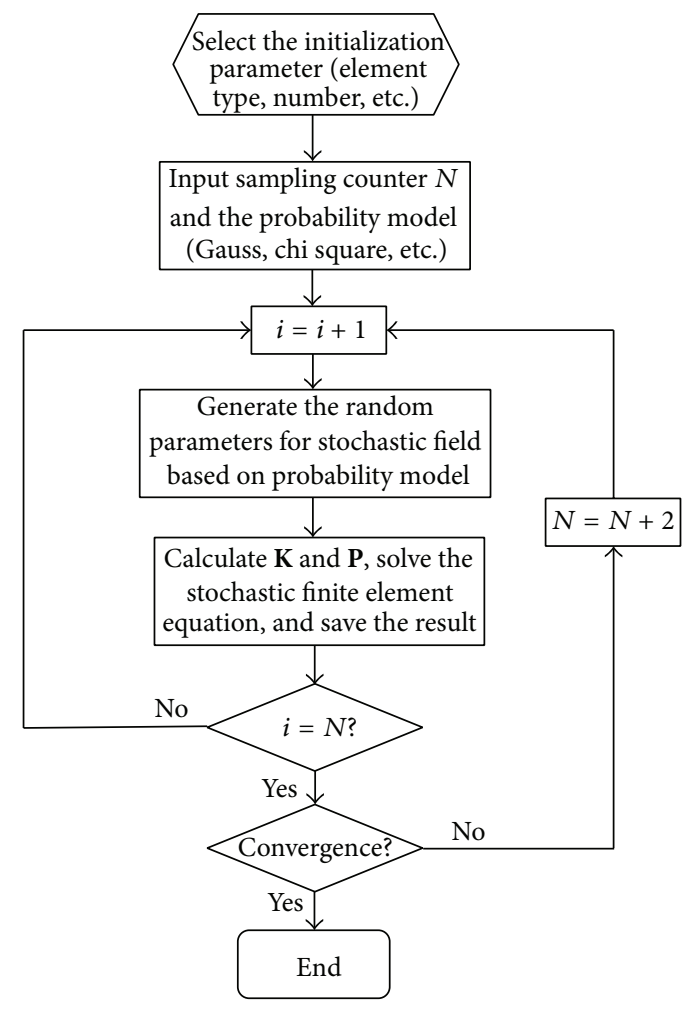

FIgURE 4: The flowchart of BSWI-SFEM.

interpolated independently, then the generalized potential energy function for the Mindlin plate bending problem is [32]

$$
\begin{aligned}
\Pi_{p}= & \iint_{\Omega} \frac{1}{2} \boldsymbol{\kappa}^{\mathrm{T}} \mathbf{D} \boldsymbol{\kappa} d x d y-\iint_{\Omega} \frac{1}{2} \gamma^{\mathrm{T}} \mathbf{C} \gamma d x d y \\
& -\iint_{\Omega} \frac{1}{2} q(x, y) w d x d y
\end{aligned}
$$

where $q$ is the concentrated load. Consider

$$
\begin{aligned}
& \boldsymbol{\kappa}=\left\{-\frac{\partial \theta_{x}}{\partial x}-\frac{\partial \theta_{y}}{\partial y}-\left(\frac{\partial \theta_{x}}{\partial y}+\frac{\partial \theta_{y}}{\partial x}\right)\right\}^{\mathrm{T}}, \\
& \mathbf{D}=\frac{E t^{3}}{12\left(1-\mu^{2}\right)}\left[\begin{array}{ccc}
1 & \mu & 0 \\
\mu & 1 & 0 \\
0 & 0 & \frac{1-\mu}{2}
\end{array}\right],
\end{aligned}
$$

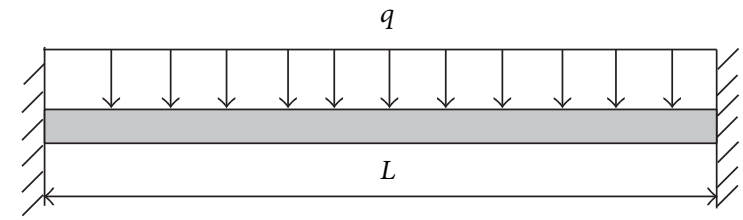

FIgure 5: The Clamp supported Timoshenko beam under a uniform load.

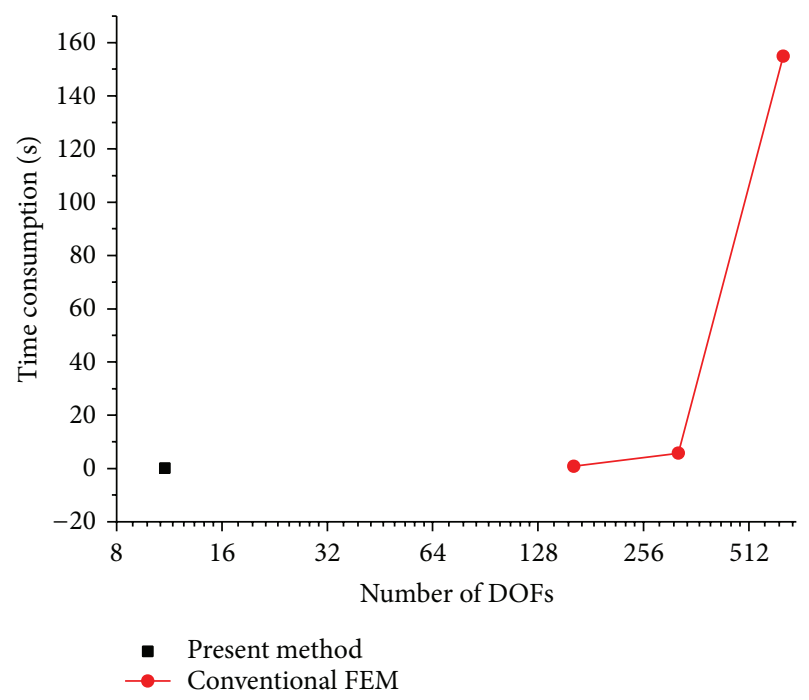

FIgURE 6: Time consumption comparison of center deflection for clamp supported beam under uniform load stochastic analysis.

$$
\begin{aligned}
& \gamma=\left\{\left(\frac{\partial w}{\partial x}-\theta_{x}\right)\left(\frac{\partial w}{\partial y}-\theta_{y}\right) 0\right\}^{\mathrm{T}}, \\
& \mathbf{C}=\frac{5 E t}{12(1+\mu)}\left[\begin{array}{lll}
1 & 0 & 0 \\
0 & 1 & 0 \\
0 & 0 & 0
\end{array}\right],
\end{aligned}
$$

where $\theta_{x}$ and $\theta_{y}$ are rotations in directions $x$ and $y, t$ is the thickness of the Mindlin plate, and $\mu$ is the Poisson ratio. Other symbols are the same as in (7). Taking BSWI scaling functions as interpolating function is as follows:

$$
w(\xi, \eta)=\boldsymbol{\Phi} \mathbf{T}^{e} \mathbf{w}^{e}, \quad \theta(\xi, \eta)=\boldsymbol{\Phi} \mathbf{T}^{e} \boldsymbol{\theta}^{e}
$$

Submitting (16) into (14), according to the generalized variational principle, the BSWI element formulation of the solving equation can be obtained as follows:

$$
\left[\begin{array}{lll}
\mathbf{K}^{e, 1} & \mathbf{K}^{e, 2} & \mathbf{K}^{e, 3} \\
\mathbf{K}^{e, 4} & \mathbf{K}^{e, 5} & \mathbf{K}^{e, 6} \\
\mathbf{K}^{e, 7} & \mathbf{K}^{e, 8} & \mathbf{K}^{e, 9}
\end{array}\right]\left[\begin{array}{l}
\mathbf{w}^{e} \\
\boldsymbol{\theta}_{x}^{e} \\
\boldsymbol{\theta}_{y}^{e}
\end{array}\right]=\left[\begin{array}{l}
\mathbf{P}^{e} \\
\mathbf{0} \\
\mathbf{0}
\end{array}\right],
$$




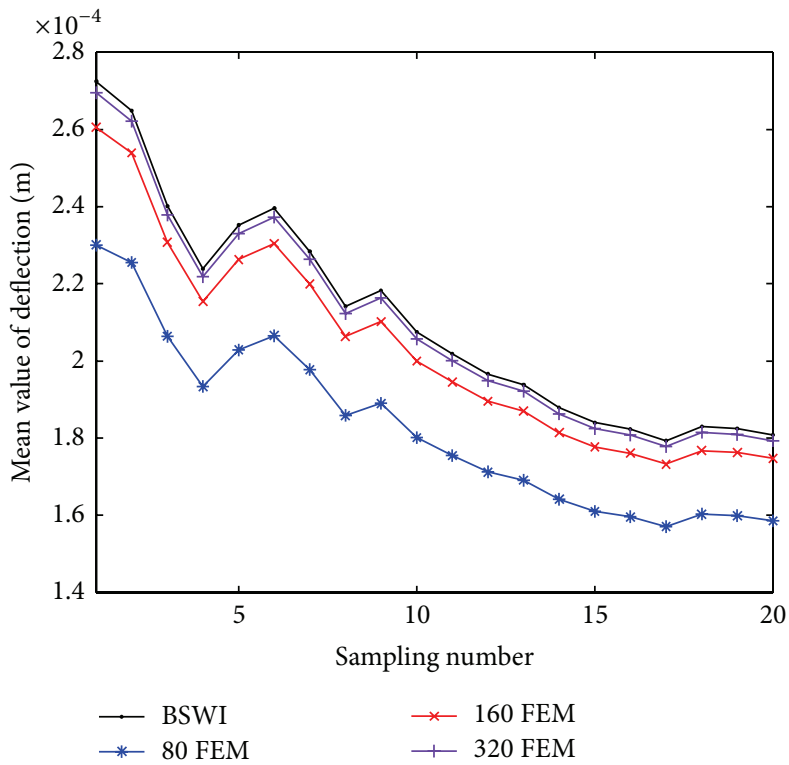

(a)

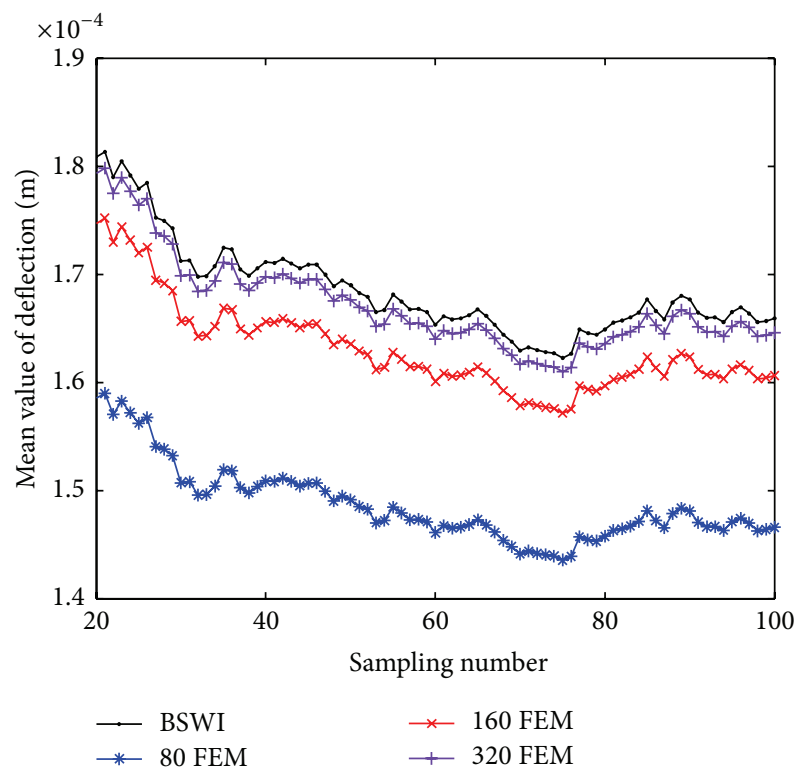

(b)

FIGURE 7: Relationship between the mean value of beam center deflection and sampling number: (a) sampling number 1-20 and (b) sampling number 20-100.

where we denote the following:

$$
\begin{gathered}
\Gamma_{1}^{0,0}=\left(\mathbf{T}_{1}^{e}\right)^{\mathrm{T}} l_{e x} \int_{0}^{1} \boldsymbol{\Phi}_{1}^{\mathrm{T}} \boldsymbol{\Phi}_{1} d \xi \mathbf{T}_{1}^{e} \\
\Gamma_{1}^{0,1}=\left(\mathbf{T}_{1}^{e}\right)^{\mathrm{T}} \int_{0}^{1} \boldsymbol{\Phi}_{1}^{\mathrm{T}} \frac{d \boldsymbol{\Phi}_{1}}{d \xi} d \xi \mathbf{T}_{1}^{e} \\
\Gamma_{1}^{1,0}=\left(\mathbf{T}_{1}^{e}\right)^{\mathrm{T}} l_{e x} \int_{0}^{1} \frac{d \boldsymbol{\Phi}_{1}^{\mathrm{T}}}{d \xi} \boldsymbol{\Phi}_{1} d \xi \mathbf{T}_{1}^{e} \\
\Gamma_{1}^{1,1}=\left(\mathbf{T}_{1}^{e}\right)^{\mathrm{T}} \frac{1}{l_{e x}} \int_{0}^{1} \frac{d \boldsymbol{\Phi}_{1}^{\mathrm{T}}}{d \xi} \frac{d \boldsymbol{\Phi}_{1}}{d \xi} d \xi \mathbf{T}_{1}^{e}
\end{gathered}
$$

$\Gamma_{2}^{i, j}(i, j=0,1)$ is similar to $\Gamma_{1}^{i, j}$ if the lengths of element in $x$-axis $l_{e x}, d \xi, \mathbf{T}_{1}^{e}$, and $\boldsymbol{\Phi}_{1}$ are replaced by $l_{e y}, d \eta, \mathbf{T}_{2}^{e}$, and $\boldsymbol{\Phi}_{2}$, respectively. Then the terms of (17) can be written as

$$
\begin{gathered}
\mathbf{K}^{e, 1}=\frac{k E t}{2(1+\mu)}\left(\Gamma_{1}^{1,1} \otimes \Gamma_{2}^{0,0}+\Gamma_{1}^{0,0} \otimes \Gamma_{2}^{1,1}\right) ; \\
\mathbf{K}^{e, 2}=-\frac{k E t}{2(1+\mu)} \Gamma_{1}^{1,0} \otimes \Gamma_{2}^{0,0} ; \\
\mathbf{K}^{e, 3}=-\frac{k E t}{2(1+\mu)} \Gamma_{1}^{0,0} \otimes \Gamma_{2}^{1,0} ; \quad \mathbf{K}^{e, 4}=\left(\mathbf{K}^{e, 2}\right)^{\mathrm{T}} ; \\
\mathbf{K}^{e, 5}=\frac{E t^{3}}{12\left(1-\mu^{2}\right)}\left\{\Gamma_{1}^{1,1} \otimes \Gamma_{2}^{0,0}+\frac{(1-\mu)}{2} \Gamma_{1}^{0,0} \otimes \Gamma_{2}^{1,1}\right\} \\
+\frac{k E t}{2(1+\mu)} \Gamma_{1}^{0,0} \otimes \Gamma_{2}^{0,0} ;
\end{gathered}
$$

$$
\begin{gathered}
\mathbf{K}^{e, 6}=\frac{E t^{3}}{12\left(1-\mu^{2}\right)}\left(\mu \boldsymbol{\Gamma}_{1}^{1,0} \otimes \Gamma_{2}^{0,1}+\frac{(1-\mu)}{2} \Gamma_{1}^{0,1} \otimes \Gamma_{2}^{1,0}\right) ; \\
\mathbf{K}^{e, 7}=\left(\mathbf{K}^{e, 3}\right)^{\mathrm{T}} ; \quad \mathbf{K}^{e, 8}=\left(\mathbf{K}^{e, 6}\right)^{\mathrm{T}} ; \\
\mathbf{K}^{e, 9}=\frac{E t^{3}}{12\left(1-\mu^{2}\right)}\left\{\Gamma_{1}^{0,0} \otimes \Gamma_{2}^{1,1}+\frac{(1-\mu)}{2} \Gamma_{1}^{1,1} \otimes \Gamma_{2}^{0,0}\right\} \\
+\frac{k E t}{2(1+\mu)} \Gamma_{1}^{0,0} \otimes \Gamma_{2}^{0,0} ; \\
\mathbf{P}^{e}=\left(\mathbf{T}^{e}\right)^{\mathrm{T}} l_{e x} l_{e y} \iint_{0}^{1} f(\xi, \eta)\left(\mathbf{\Phi}_{1} \otimes \mathbf{\Phi}_{2}\right)^{\mathrm{T}} d \xi d \eta .
\end{gathered}
$$

The element solving equation of vibration problem is

$$
|\mathbf{K}-\lambda \mathbf{M}|=0,
$$

where $\mathbf{M}$ is the following consistent mass matrix:

$$
\mathbf{M}=\rho l_{e x} l_{e y} t\left(\mathbf{T}^{e}\right)^{\mathrm{T}}\left(\frac{d \boldsymbol{\Phi}^{\mathrm{T}}}{d \xi}\right) \otimes\left(\frac{d \boldsymbol{\Phi}}{d \eta}\right) \mathbf{T}^{e} .
$$

$\mathbf{K}$ is the following stiffness matrix:

$$
\mathbf{K}=\left[\begin{array}{lll}
\mathbf{K}^{e, 1} & \mathbf{K}^{e, 2} & \mathbf{K}^{e, 3} \\
\mathbf{K}^{e, 4} & \mathbf{K}^{e, 5} & \mathbf{K}^{e, 6} \\
\mathbf{K}^{e, 7} & \mathbf{K}^{e, 8} & \mathbf{K}^{e, 9}
\end{array}\right]
$$

$\rho$ is density and $\lambda$ is the vibration engine value. 


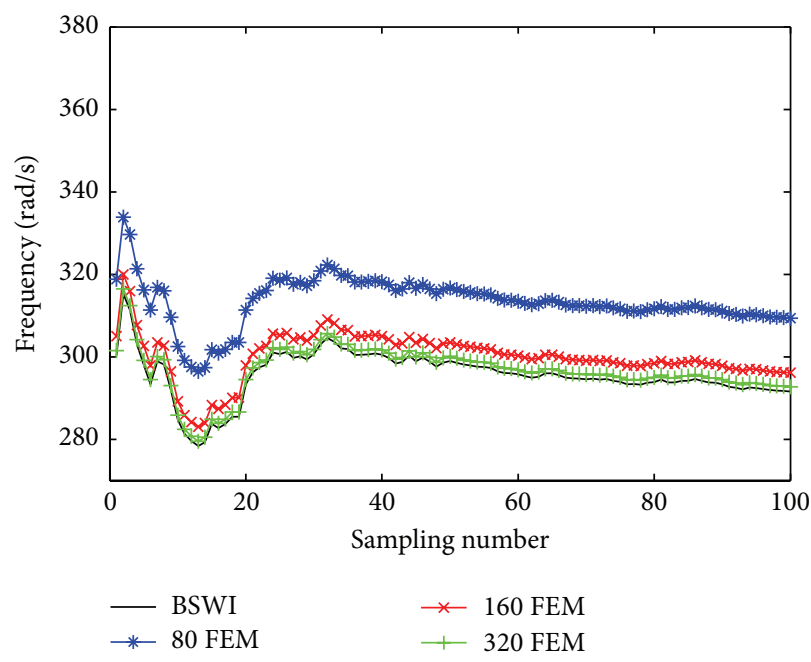

(a)

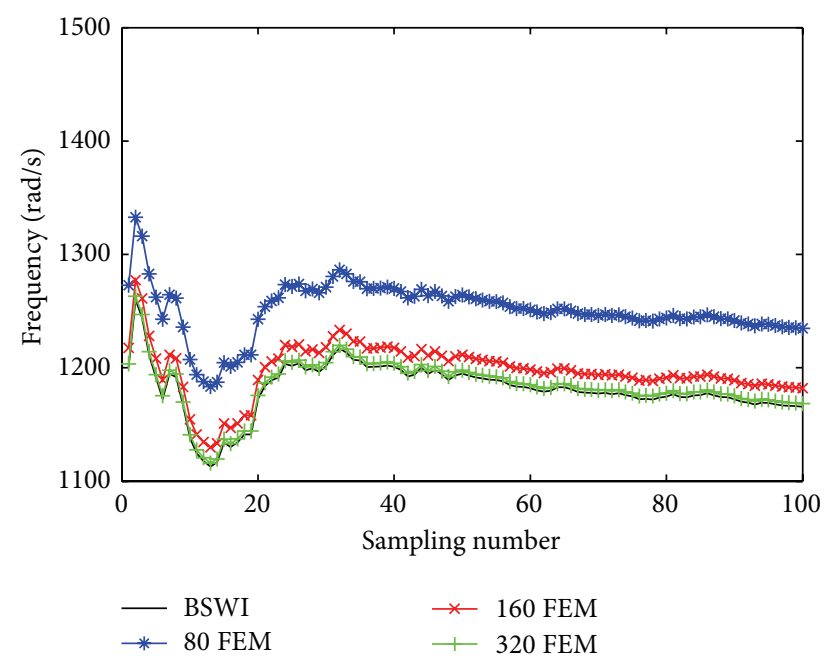

(b)

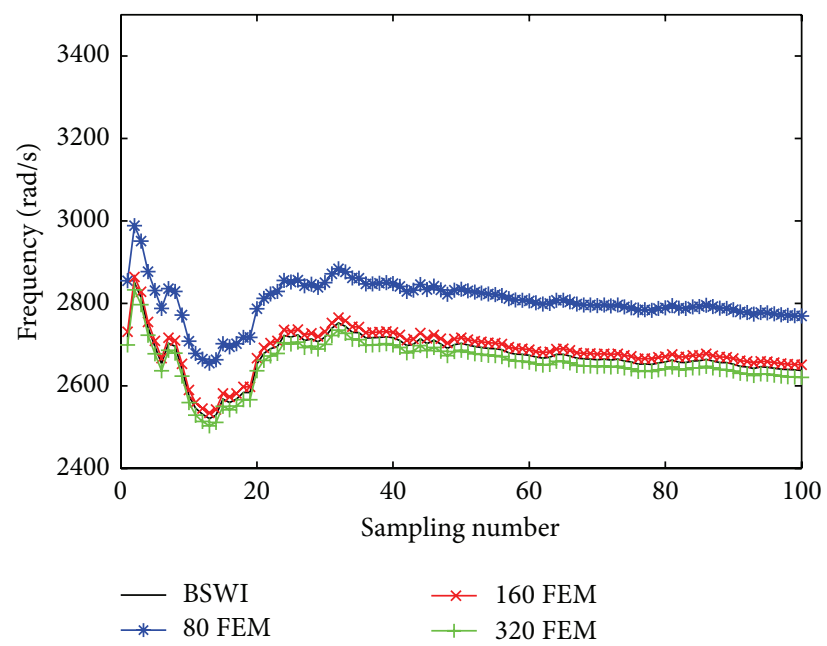

(c)

FIGURE 8: Relationship between the first three natural frequencies and sampling numbers of the Timoshenko beam. (a) First-order circular frequency, (b) second-order circular frequency, and (c) third-order circular frequency.

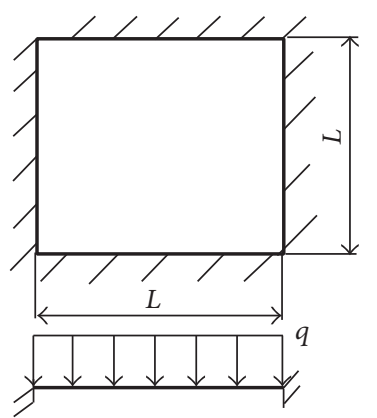

(a)

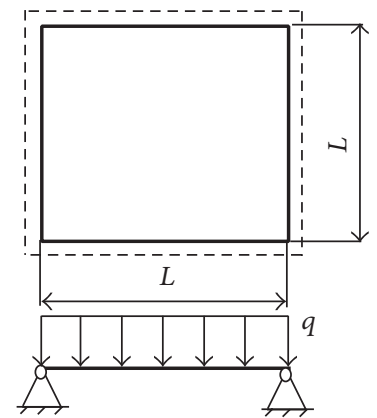

(b)

Figure 9: The Clamp and simply supported Mindlin plate under a uniform load: (a) clamp supported and (b) simply supported. 
2.5. BSWI Plane Rigid Frame Element. The BSWI plane rigid frame element can be seen as the simple superposition of the axial rod element and the Euler beam element. The displacement of element nodes, solving domain, and corresponding coordinates are shown in Figure 3.
It can be seen from Figure 3 that there are $2^{j+1}+4$ DOFs of one element. The physical DOFs of BSWI plane rigid element in local coordinate $(X, Y)$ are

$$
\boldsymbol{\delta}^{e}=\left(\begin{array}{lllllllllllll}
u_{1} & w_{1} & M_{1} & u_{2} & w_{2} & M_{2} & \cdots & u_{n-1} & w_{n-1} & M_{n-1} & u_{n} & w_{n} & M_{n}
\end{array}\right)^{\mathrm{T}},
$$

while the corresponding DOFs in global coordinate $(\bar{X}, \bar{Y})$ are

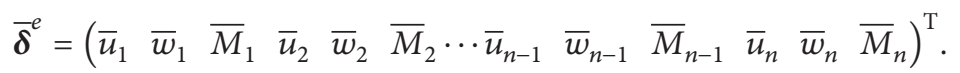

In order to construct the BSWI plane rigid frame element, the relationship between global and local coordinates should be obtained. Consider

$$
\left[\begin{array}{c}
u_{i} \\
w_{i} \\
M_{i}
\end{array}\right]=\mathbf{g}_{i}\left[\begin{array}{c}
\bar{u}_{i} \\
\bar{w}_{i} \\
M_{i}
\end{array}\right], \quad i=1, \ldots, n,
$$

where

$$
\mathbf{g}_{i}=\left[\begin{array}{ccc}
\cos \alpha & \sin \alpha & 0 \\
-\sin \alpha & \cos \alpha & 0 \\
0 & 0 & 1
\end{array}\right], \quad i=1, \ldots, n
$$

Therefore,

$$
\boldsymbol{\delta}^{e}=\mathbf{G}^{e} \overline{\boldsymbol{\delta}}^{e}
$$

Therefore, the BSWI plane rigid frame element for bending problem analysis in global coordinate is

$$
\overline{\mathbf{K}} \overline{\boldsymbol{\delta}}=\overline{\mathbf{P}} .
$$

The BSWI plane rigid frame element for vibration problem analysis in global coordinate is

$$
|\overline{\mathbf{K}}-\lambda \overline{\mathbf{M}}|=0 \text {. }
$$

Then, by introducing the BSWI axial rod element and the BSWI Euler beam element [40], the BSWI plane rigid frame can be obtained.

\section{Stochastic Finite Element Based on B-Spline Wavelet on the Interval}

The application of the MCS-FEM to large-scale structures was impossible due to its excessive computation cost a few years ago due to the huge calculation amount. Let us consider the event that the random variable $x$ can take the discrete values $x=1, \ldots, N$ with probabilities $p_{1}, p_{2}, \ldots, p_{N}$, respectively.
The corresponding probability distribution functions can be expressed as

$$
p(x)=\sum_{i=1}^{N} p_{i} \delta(x-i)
$$

where $\delta(x)$ is the Dirac function, and the corresponding cumulative distribution function can be expressed as

$$
P(x)= \begin{cases}0, & \text { if } j<0, \\ \sum_{i=1}^{j} p_{j}, & \text { if } 1 \leq j \leq N \\ 1, & \text { if } j>1 .\end{cases}
$$

Since a discrete distribution is used to approximate the continuous distribution in the real events, the sampling counter $N$ should be big enough.

While BSWI-FEM has the property of high accuracy and efficiency [26], through inosculating this technology with MCS, the limitation of applying MCS-FEM to large-scale structures can be alleviated further.

Therefore, the stochastic wavelet finite element based on B-spline wavelet on the interval is proposed by the aid of combining Monte Carlo method with BSWI-FEM. The first step is to select the initialization parameters, such as element type and element number. Then the sampling counter $N$ should be decided, and the probability model of variables also needs to be selected. With these inputs, the calculation of BSWI-SFEM can be started as shown in Figure 4. Then the stiffness matrix $\mathbf{K}$ and the force vector $\mathbf{P}$ are calculated by BSWI-SFEM. Finally, through BSWI-SFEM, the results can be obtained. This process will be repeated again and again until converged. Considering the robustness of the procedure, the sampling counter $N$ is set as a variable in course; this can guarantee the convergence. 


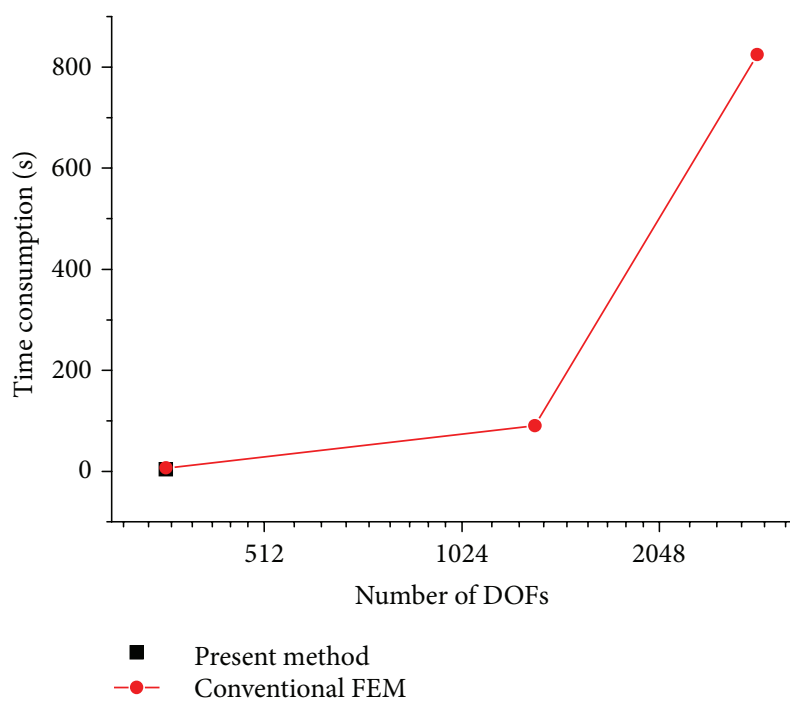

FIGURE 10: Time consumption comparison of center deflection for the clamp supported Mindlin plate under uniform load stochastic analysis.

\section{Numerical Examples}

To stochastic analysis problem, it is hard to define an exact solution. But in one sense, the solution obtained by a large number of samples could be a good approximation of the real exact solution. In this paper, we use this kind of sample exact solution as an index to evaluate the property of the constructed BSWI-FEM. Furthermore, we will give a traditional result of finite element analysis before the BSWI-FEM analysis to validate the accuracy of the proposed method.

All the programs are implemented in the MATLAB environment. In all stochastic simulations, a computer with a $2.5 \mathrm{GHz}$ Intel $\mathrm{CPU}$ and 2 gigabytes of memory is used, and the operating system is 32 -bit Windows XP. We assume that all the parameters obey the normal distribution: the mean value of length is $1000 \mathrm{~mm}(1000 \mathrm{~mm} \times 1000 \mathrm{~mm}$ when it is plate), the mean value of the cross section for beam is $20 \mathrm{~mm}$ $\times 12 \mathrm{~mm}$, the mean value of plate thickness is $10 \mathrm{~mm}$, the mean value amplitude of load is $100 \mathrm{~N}$, the mean value of density is $7917 \mathrm{~kg} / \mathrm{m}^{3}$, and the mean value of Young's modulus is $206 \mathrm{MPa}$. The standard deviations of these parameters are $5 \%$ of their mean values.

4.1. Bending of the Timoshenko Beam. A clamp supported Timoshenko beam under a uniform load (as shown in Figure 5) is considered in this example. The parameters are mentioned above. It is demonstrated in Table 1 that the proposed BSWI element has a good agreement with the exact solution [38].

4.2. Stochastic Analysis of the Timoshenko Beam Bending. A stochastic analysis for a clamp supported Timoshenko beam under a uniform load as shown in Figure 5 is considered in this numerical example. The results of BSWI-SFEM are shown in Table 2 compared with conventional FEM. It is

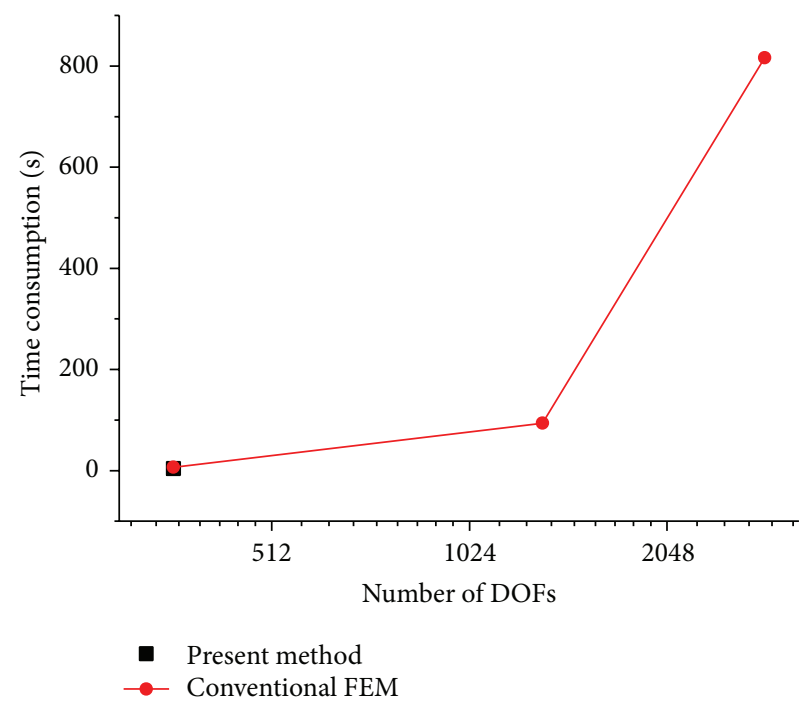

FIgURE 11: Time consumption comparison of center deflection for the simply supported Mindlin plate under uniform load stochastic analysis.

TABLE 1: Comparison of center deflection of clamp supported beam under uniform load.

\begin{tabular}{lc}
\hline Mesh method (DOFs) & Center deflection $[\mathrm{mm}]$ \\
\hline 80 traditional FEM (162) & 0.14114 \\
160 traditional FEM (322) & 0.15399 \\
320 traditional FEM (642) & 0.15758 \\
1 BSWI FEM (11) & 0.15876 \\
Exact solution [38] & 0.15802 \\
\hline
\end{tabular}

TABLE 2: Comparison of center deflection of clamp supported beam under uniform load (stochastic analysis).

\begin{tabular}{lcc}
\hline Mesh method (DOFs) & $\begin{array}{c}\text { Center deflection } \\
{[\mathrm{mm}]}\end{array}$ & $\begin{array}{c}\text { Time consumption } \\
{[\mathrm{s}]}\end{array}$ \\
\hline 80 traditional FEM (162) & 0.14660 & 0.781000 \\
160 traditional FEM (322) & 0.16066 & 5.765000 \\
320 traditional FEM (642) & 0.16462 & 154.875000 \\
1 BSWI FEM (11) & 0.16593 & 0.078000 \\
Sample exact solution & 0.16368 & - \\
\hline
\end{tabular}

evident that 1 BSWI-SFEM element can achieve a similar accuracy as 320 conventional Timoshenko elements. However, the proposed method only needs $0.078 \mathrm{~s}$ to establish the computation, while the conventional method needs $154.875 \mathrm{~s}$ as shown in Figure 6.

Figure 7 presents the mean value of deflection at the center of beam which varies with the counter of sample. It can be seen that 1 BSWI-SFEM element obtains a good approximation of the center deflection compared with conventional method. Although the conventional method could approximate the sample exact solution with mesh refinement, the time consumption increases sharply. This kind of increase would result in unacceptable time consumption for the conventional method when it is used in large-scale structures. 


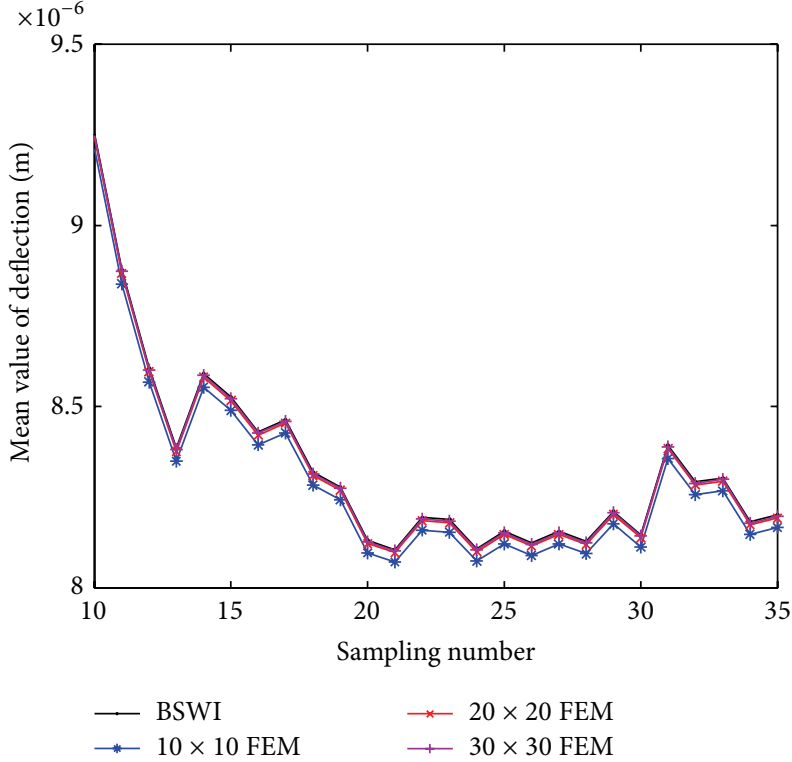

(a)

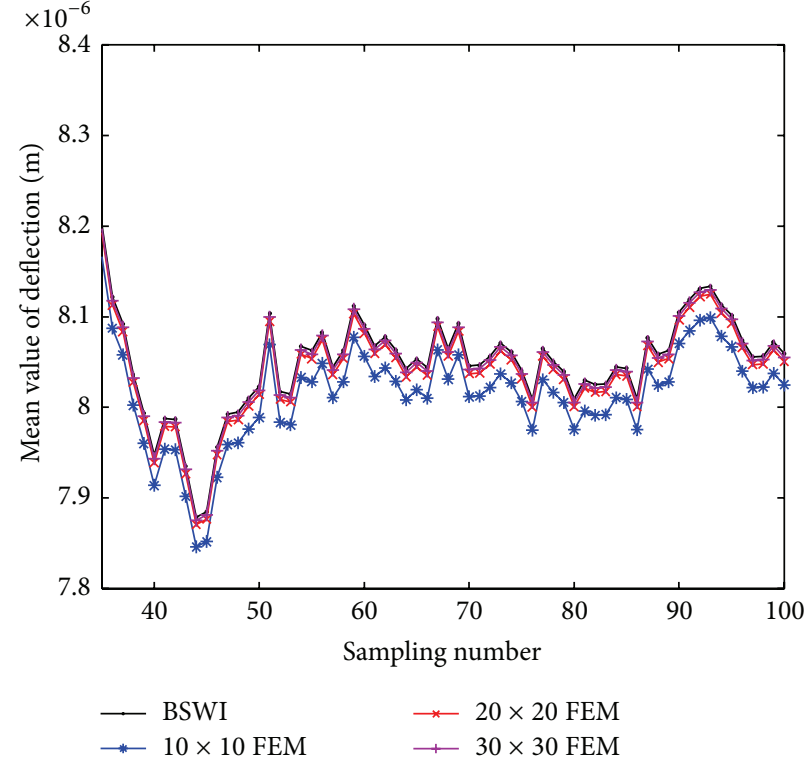

(b)

FIGURE 12: Relationship between the mean value of plate center deflection and sampling number: (a) sampling number 10-35, (b) sampling number 35-100.

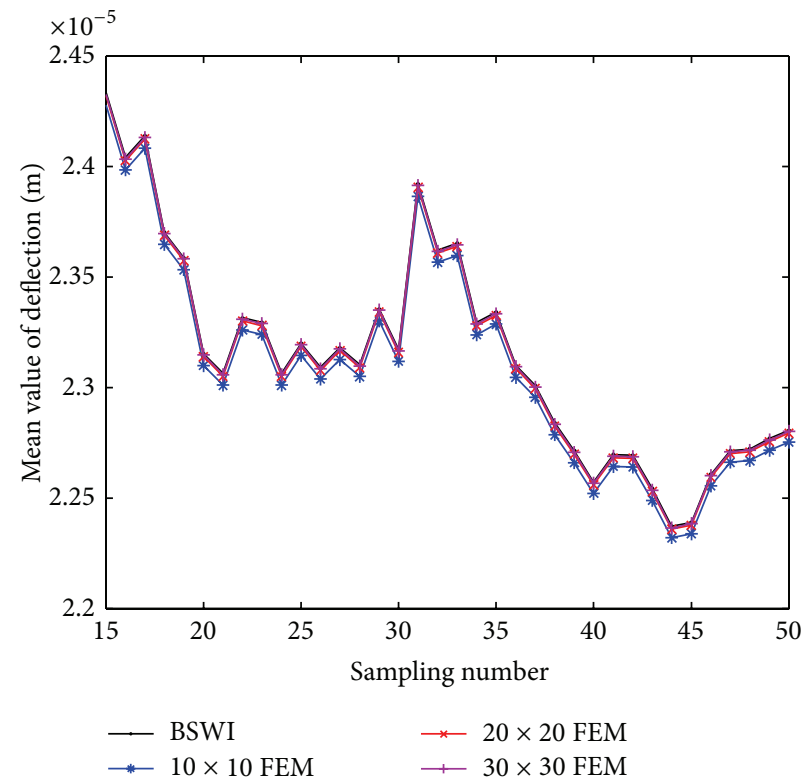

(a)

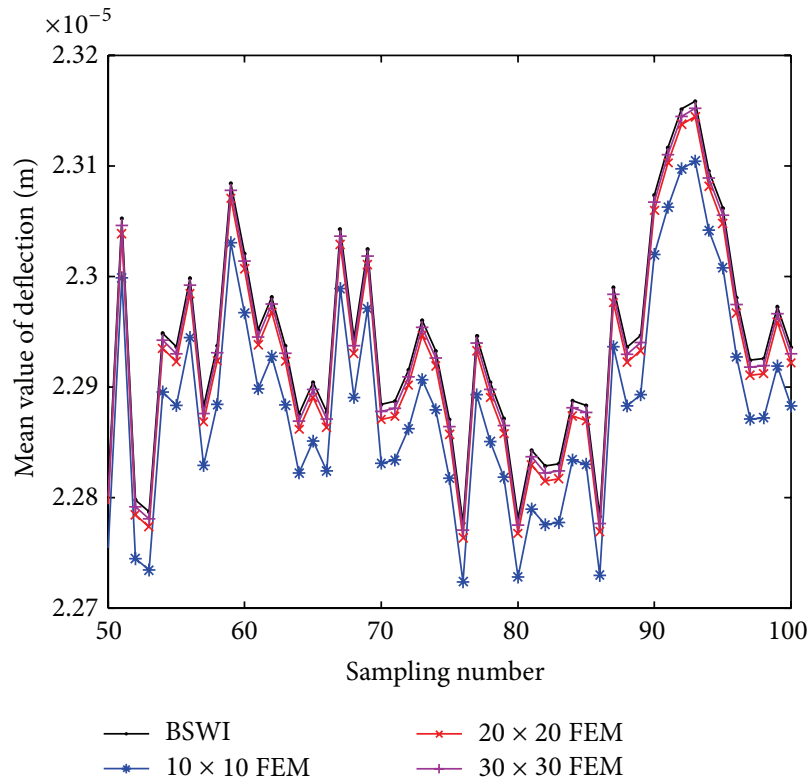

(b)

FIGURE 13: Relationship between the mean value of plate center deflection and sampling number: (a) sampling number 15-50, (b) sampling number 50-100.

However, owing to the efficiency and accuracy of BSWISFEM, this problem can be greatly eased.

4.3. Stochastic Analysis of the Timoshenko Beam Vibration. Figure 8 shows the vibration analysis results of the clamp supported Timoshenko beam. BSWI results are compared with traditional finite element method. It can be seen that 1 BSWI-SFEM element can obtain a good approximation of natural frequencies compared with traditional method. Although the conventional method could approximate the sample exact solution with mesh refinement, the time consumption increases sharply. In this numerical example, 


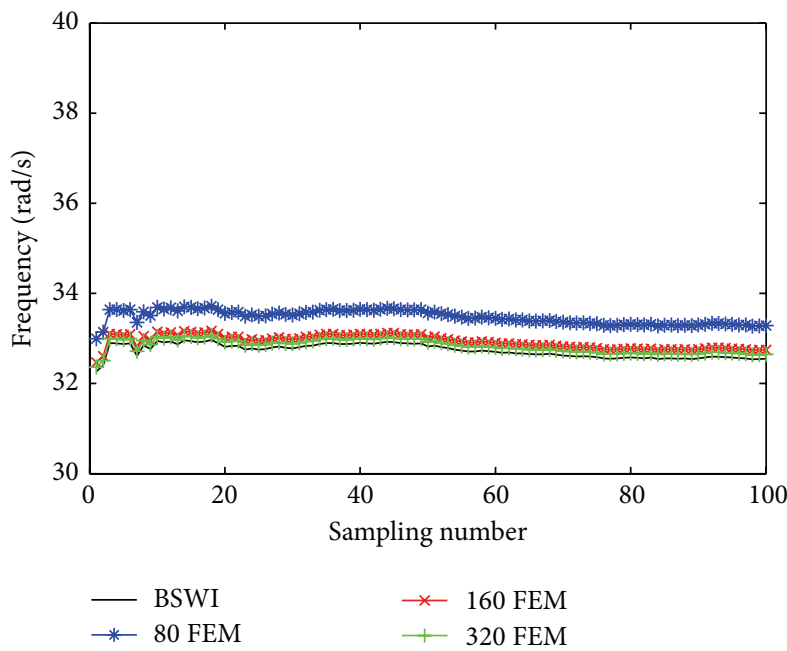

(a)

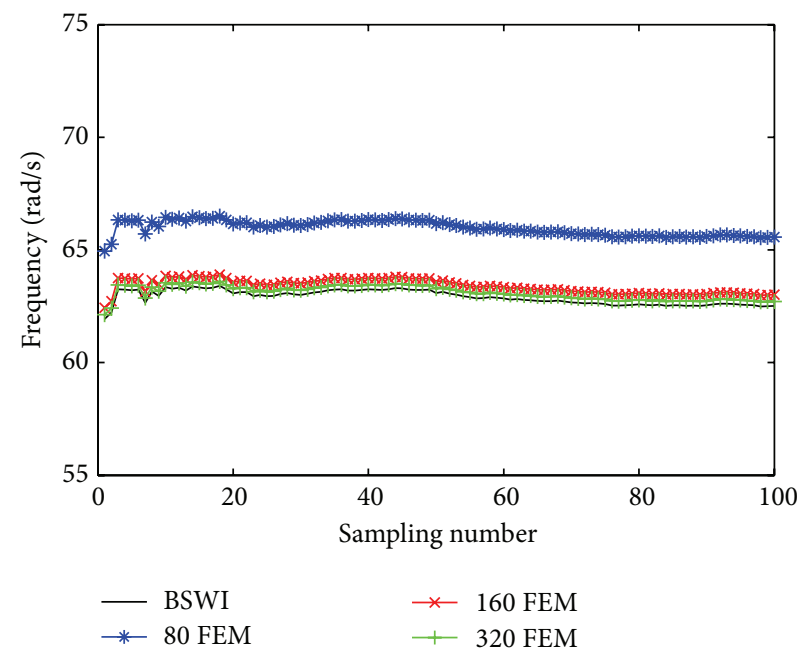

(b)

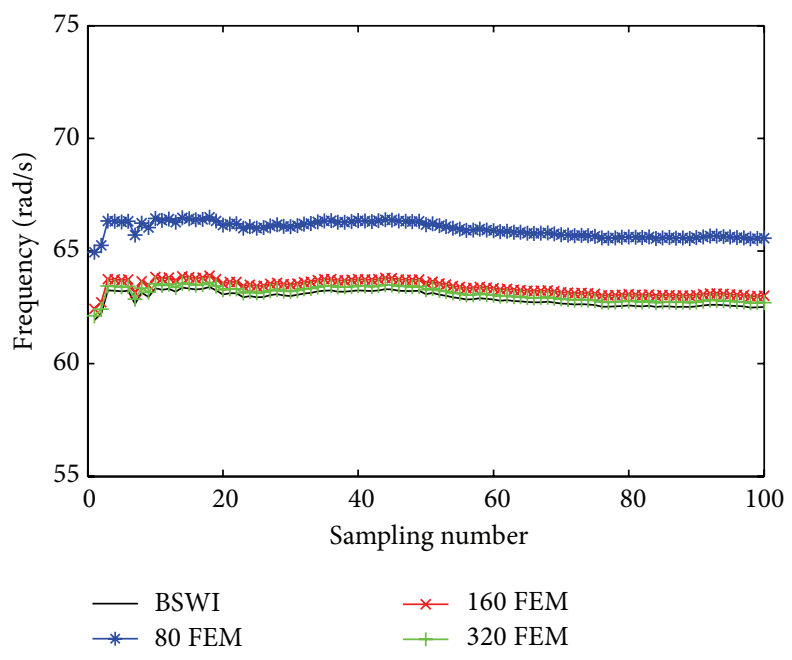

(c)

FIGURE 14: Relationship between the first three natural frequencies and sampling numbers of the Mindlin plate. (a) First-order circular frequency, (b) second-order circular frequency, and (c) third-order circular frequency.

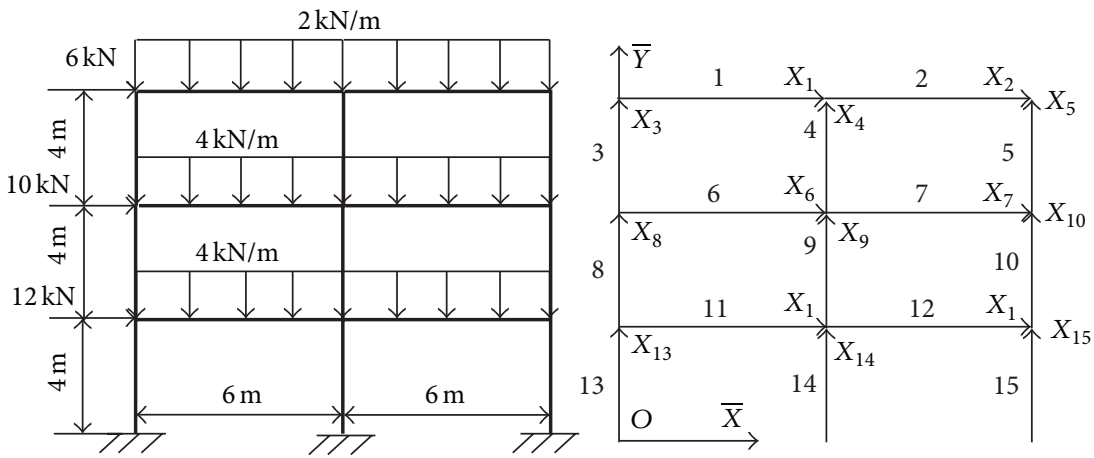

FIGURE 15: Coordinates, material parameters, and load parameters of plane rigid frame. 


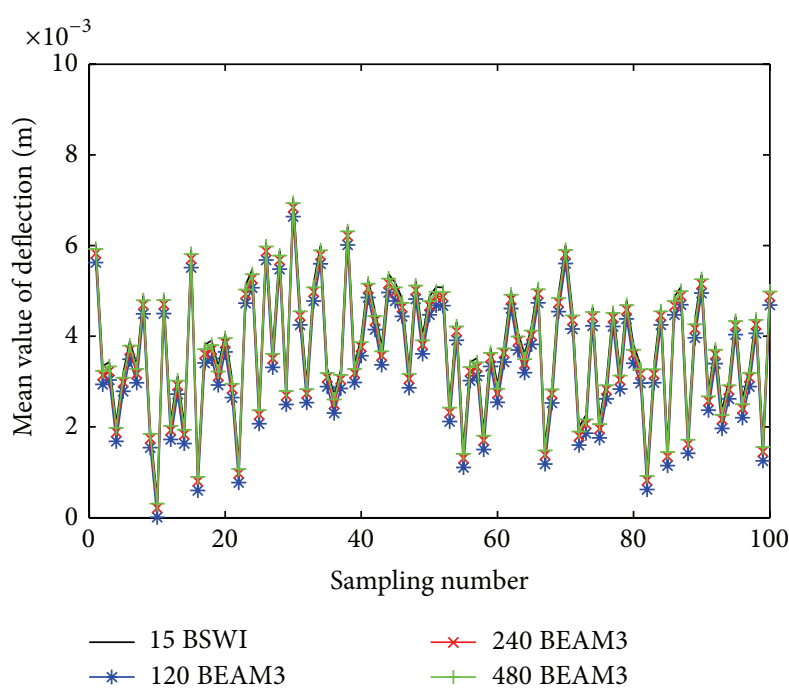

(a)

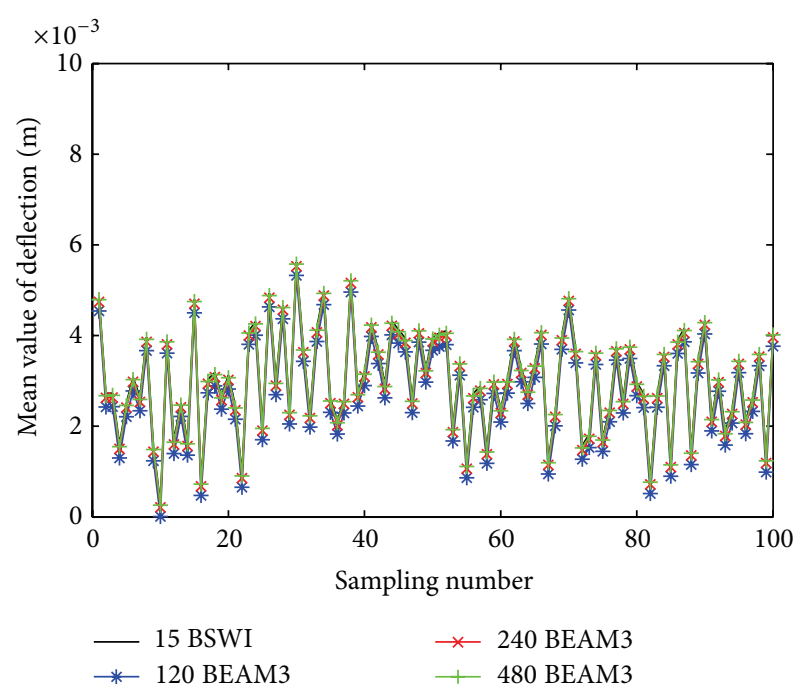

(b)

FIGURE 16: Relationship between the bending analysis results and sampling number of the plane rigid frame. (a) Point A and (b) point B.

TABLE 3: Comparison of center deflection of the clamp and simply supported Mindlin plate under uniform load.

\begin{tabular}{lcc}
\hline & \multicolumn{2}{c}{ Center deflection $[\mu \mathrm{m}]$} \\
Mesh method (DOFs) & $\begin{array}{c}\text { The clamp } \\
\text { supported Mindlin } \\
\text { plate }\end{array}$ & $\begin{array}{c}\text { The simply } \\
\text { supported Mindlin } \\
\text { plate }\end{array}$ \\
\hline 100 traditional FEM (363) & 7.9426 & 22.598 \\
400 traditional FEM (1323) & 7.9676 & 22.637 \\
900 traditional FEM (2883) & 7.9722 & 22.644 \\
1 BSWI FEM (363) & 7.9761 & 22.651 \\
Exact solution [35] & 7.9760 & 22.649 \\
\hline
\end{tabular}

the proposed BSWI method only needs $6.005 \mathrm{~s}$ to establish the computation with 1 element, while the conventional method needs $240.758 \mathrm{~s}$ to obtain the results in a similar accuracy with 320 elements.

4.4. Bending of the Mindlin Plate. In this part, the Mindlin plate bending problem as presented in Figure 9 is used to test the accuracy of the BSWI Mindlin plate element. The corresponding parameters are chosen as the mean value of these stochastic parameters mentioned above. The numerical results given in Table 3 indicate that the proposed method has a good agreement with the exact solution [38] once more.

4.5. Stochastic Analysis of the Mindlin Plate Bending. Stochastic analysis of a square Mindlin plate with clamp and simply supported boundary conditions subjected to a uniform load as shown in Figure 9 is considered in this numerical example. The stochastic bending analysis of this plate is carried out by BSWI-SFEM incorporated with the Monte Carlo simulation. The center deflection of the two kinds of boundary conditions is presented in Tables 4 and 5. The results show
TABLE 4: Comparison of center deflection of the clamp supported Mindlin plate under uniform load (stochastic analysis).

\begin{tabular}{lcc}
\hline Mesh method (DOFs) & $\begin{array}{c}\text { Center deflection } \\
{[\mu \mathrm{m}]}\end{array}$ & $\begin{array}{c}\text { Time consumption } \\
{[\mathrm{s}]}\end{array}$ \\
\hline 100 traditional FEM (363) & 8.0250 & 6.766000 \\
400 traditional FEM (1323) & 8.0506 & 90.562000 \\
900 traditional FEM (2883) & 8.0553 & 824.875000 \\
1 BSWI FEM (363) & 8.0592 & 4.015000 \\
Sample exact solution & 8.0590 & - \\
\hline
\end{tabular}

TABLE 5: Comparison of center deflection of the simply supported Mindlin plate under uniform load (stochastic analysis).

\begin{tabular}{lcc}
\hline Mesh method (DOFs) & $\begin{array}{c}\text { Center deflection } \\
{[\mu \mathrm{m}]}\end{array}$ & $\begin{array}{c}\text { Time consumption } \\
{[\mathrm{s}]}\end{array}$ \\
\hline 100 traditional FEM (363) & 22.883 & 6.821000 \\
400 traditional FEM (1323) & 22.922 & 93.610000 \\
900 traditional FEM (2883) & 22.930 & 816.320000 \\
1 BSWI FEM (363) & 22.936 & 3.986000 \\
Sample exact solution & 22.934 & - \\
\hline
\end{tabular}

that the BSWI-SFEM can give a better approximation for the sample exact solution compared with the conventional method. Furthermore, the time consumption as shown in Figures 10 and 11 indicates that BSWI-SFEM is more efficient than the conventional method, which allows the proposed approach to be used for the large-scale structures. The further comparisons are presented in Figures 12 and 13. Using the mean value of output variables, the reliability calculations can be thereafter performed.

4.6. Stochastic Analysis of the Mindlin Plate Vibration. The vibration analysis results of the clamp supported Mindlin 


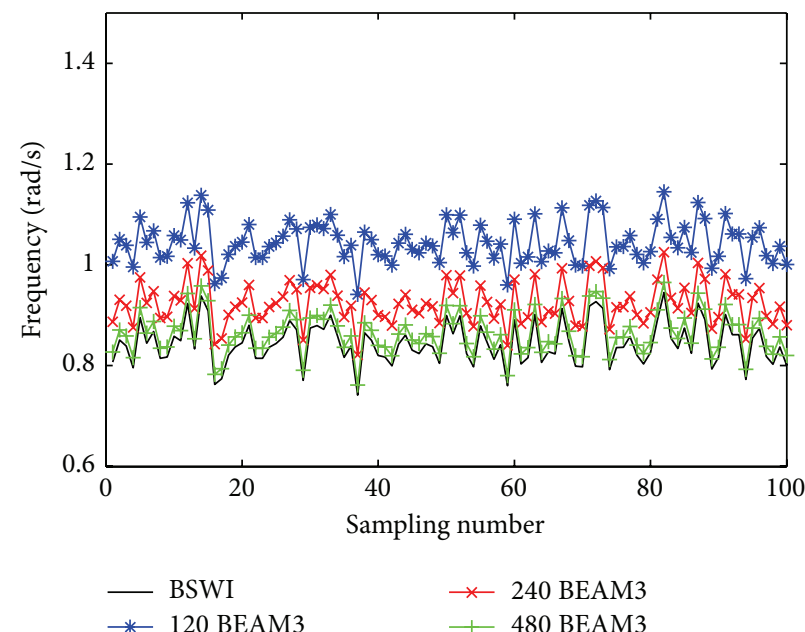

(a)

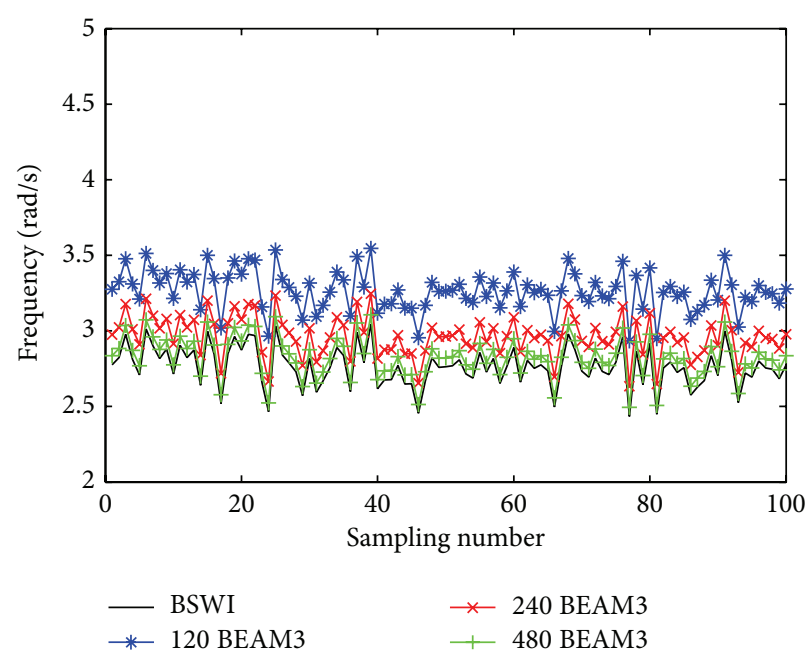

(b)

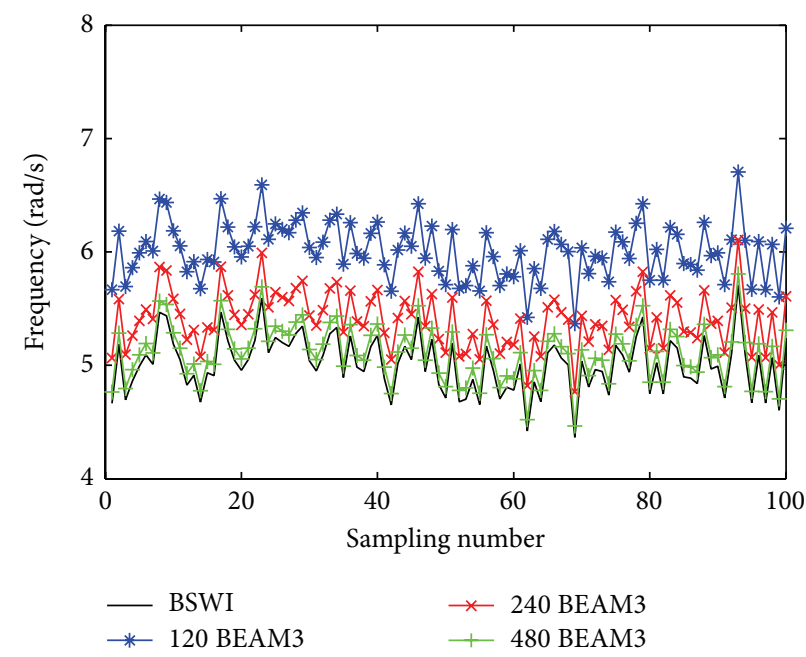

(c)

FIGURE 17: Relationship between the first three natural frequencies and sampling numbers of plane rigid frame. (a) First-order circular frequency, (b) second-order circular frequency, and (c) third-order circular frequency.

plate are shown in Figure 14. The first three natural frequency results of BSWI element are compared with conventional element. It can be seen from the results that 1 BSWI element can obtain the results in a similar accuracy with 320 traditional elements. However, 1 BSWI method only needs $10.397 \mathrm{~s}$ to establish the computation, while the conventional method needs $4320.385 \mathrm{~s}$ to obtain the results in a similar accuracy with 320 elements.

4.7. Stochastic Analysis of Plane Rigid Frame Bending. As shown in Figure 15, the plane rigid frame combined by 15 components is considered in this example. We assume that all the parameters obey the normal distribution. The corresponding material parameters are elastic modulus $E=$ $2.06 \times 10^{11} \mathrm{~N} / \mathrm{m}^{2}$, width $b=0.012 \mathrm{~m}$, height $h=0.012 \mathrm{~m}$, and density $\rho=7917 \mathrm{~kg} / \mathrm{m}^{3}$, respectively. The standard deviations of these parameters are $5 \%$ of their mean values.
As shown in Figure 16, the bending analysis results of BSWI element on points A and B are compared with BEAM3 element. Although BEAM3 element can obtain the results with high accuracy by grid refinement, it will spend much more time on computation. However, the same solving domain of plane rigid frame is discreted by only 15 BSWI elements; it can achieve the results in a similar accuracy with 480 BEAM3 elements. So the efficiency and solving precision of BSWI-SFEM plane rigid element are verified.

4.8. Stochastic Analysis of Plane Rigid Frame Vibration. The stochastic vibration analysis of plane rigid frame is carried out by BSWI-SFEM incorporated with the Monte Carlo simulation in this example. The corresponding analysis results are shown in Figure 17. BSWI element results are compared with BEAM3 element. It can be seen from the comparison that, although BEAM3 element can acquire the results with high 
accuracy by grid refinement, much more time is spent. BSWISFEM is superior, with only 15 elements, and the results with high efficiency and accuracy can be achieved.

\section{Conclusions}

Based on B-spline wavelet on the interval and the Monte Carlo simulation, the stochastic finite element method is developed in the paper. The proposed method can deal with the response variability resulting from the spatial variability of the structural material properties. Taking the Timoshenko beam and the Mindlin plate as examples, the corresponding model are constructed and solved. The numerical examples illustrate that the proposed method can achieve a high analytical accuracy and efficient convergence in solving the stochastic problems. Although only three kinds of stochastic BSWI elements are constructed in the paper, further extensive work is easy to be established for solving the buckling and vibration problems of structures based on the same interpolation functions and approaches. The BSWI-SFEM is feasible to carry out the reliability analysis of large-scale engineering structures.

\section{Conflict of Interests}

The authors declare that there is no conflict of interests regarding the publication of this paper.

\section{Acknowledgments}

Special thanks should be expressed to the editor and the referees. This work was supported by the National Natural Science Foundation of China (nos. 51335006 and 51225501), the Project funded by China Postdoctoral Science Foundation (no. 2014M552432), the Fundamental Research for the Central Universities, the National Science and Technology Major Project of China (no. 2012ZX04002071), and the Program for Changjiang Scholars and Innovative Research Team in University.

\section{References}

[1] R. N. Jazar, Vehicle Dynamics: Theory and Application, Springer, 2008.

[2] H. R. Hamidzadeh and R. N. Jazar, Vibrations of Thick Cylindrical Structures, Springer, 2010.

[3] S. S. Ramesh, K. M. Lim, and B. C. Khoo, "An axisymmetric hypersingular boundary integral formulation for simulating acoustic wave propagation in supercavitating flows," Journal of Sound and Vibration, vol. 331, pp. 4313-4342, 2012.

[4] X. F. Wu, Y. J. Lei, D. K. Li et al., "Transfer function modeling of structural vibration of complex aerospace structures based on finite element analysis," Journal of Mechanical Science and Technology, vol. 27, pp. 1245-1253, 2013.

[5] R. Mellah, G. Auvinet, and F. Masrouri, "Stochastic finite element method applied to non-linear analysis of embankments," Probabilistic Engineering Mechanics, vol. 15, no. 3, pp. 251-259, 2000.
[6] G. I. Schuëller, "Computational stochastic mechanics-recent advances," Computers and Structures, vol. 79, no. 22-25, pp. 2225-2234, 2001.

[7] S. Chakraborty and S. S. Dey, "Stochastic finite element simulation of uncertain structures subjected to earthquake," Shock and Vibration, vol. 7, no. 5, pp. 309-320, 2000.

[8] A. C. Cornell, "First order uncertainty analysis of soils deformation and stability," in Proceedings of the 1st International Conference on Applications of Statistics and Probability to Soil and Structural Engineering, Hong Kong, China, 1970.

[9] M. Kamiński, "Generalized perturbation-based stochastic finite element method in elastostatics," Computers and Structures, vol. 85, no. 10, pp. 586-594, 2007.

[10] X. G. Hua, Y. Q. Ni, Z. Q. Chen, and J. M. Ko, "An improved perturbation method for stochastic finite element model updating," International Journal for Numerical Methods in Engineering, vol. 73, no. 13, pp. 1845-1864, 2008.

[11] M. A. Valdebenito, A. A. Labarca, and H. A. Jensen, "On the application of intervening variables for stochastic finite element analysis," Computers \& Structures, vol. 126, pp. 164-176, 2013.

[12] J. Zhang and B. Ellingwood, "Orthogonal series expansions of random fields in reliability analysis," Journal of Engineering Mechanics, vol. 120, no. 12, pp. 2660-2677, 1994.

[13] S. Sakamoto and R. Ghanem, "Polynomial chaos decomposition for the simulation of non-Gaussian nonstationary stochastic processes," Journal of Engineering Mechanics, vol. 128, no. 2, pp. 190-201, 2002.

[14] C. Proppe, "Multiresolution analysis for stochastic finite element problems with wavelet-based Karhunen-Loeve expansion," Mathematical Problems in Engineering, vol. 2012, Article ID 215109, 15 pages, 2012.

[15] L. F. Yang, Y. E. Zhou, J. J. Zhou et al., "Hierarchical stochastic finite element method for structural analysis," Acta Mechanica Solida Sinica, vol. 26, no. 2, pp. 189-196, 2013.

[16] N. Metropolis and S. Ulam, "The Monte Carlo method," Journal of the American Statistical Association, vol. 44, no. 247, pp. 335341, 1949.

[17] J.-G. Han, W.-X. Ren, and Y. Huang, "A wavelet-based stochastic finite element method of thin plate bending," Applied Mathematical Modelling, vol. 31, no. 2, pp. 181-193, 2007.

[18] F. A. Diazdelao and S. Adhikari, "Gaussian process emulators for the stochastic finite element method," International Journal for Numerical Methods in Engineering, vol. 87, no. 6, pp. 521-540, 2011.

[19] S. H. Han and M. S. Bang, "Probabilistic optimal safety valuation based on stochastic finite element analysis of steel cablestayed bridges," Smart Structures and Systems, vol. 10, no. 2, pp. 89-110, 2012.

[20] C. Lang, A. Doontan, and K. Maute, "Extended stochastic FEM for diffusion problems with uncertain material interfaces," Computational Mechanics, vol. 51, no. 6, pp. 1031-1049, 2013.

[21] F. Augustin and P. Rentrop, "Stochastic Galerkin techniques for random ordinary differential equations," Numerische Mathematik, vol. 122, no. 3, pp. 399-419, 2012.

[22] S. Shang and G. J. Yun, "Stochastic finite element with material uncertainties: implementation in a general purpose simulation program," Finite Elements in Analysis and Design, vol. 64, pp. 65-78, 2013.

[23] F. Salvat, J. M. Fernández-Varea, and J. Sempau, PENELOPE 2008: A Code System for Monte Carlo Simulation of Electron and Photon Transport, Universitat de Barcelona, Barcelona, Spain, 2008. 
[24] G. I. Schuëller, "Developments in stochastic structural mechanics," Archive of Applied Mechanics, vol. 75, pp. 755-773, 2006.

[25] S. G. Mallat, A Wavelet Tour of Signal Processing, Academic Press, London, UK, 1999.

[26] A. Cohen, Numerical Analysis of Wavelet Method, Elsevier Press, Amsterdam, The Netherlands, 2003.

[27] V. Vampa, M. T. Martin, and E. Serrano, "A hybrid method using wavelets for the numerical solution of boundary value problems on the interval," Applied Mathematics and Computation, vol. 217, no. 7, pp. 3355-3367, 2010.

[28] S. S. Ray and A. Dandapat, "Wavelet-Galerkin method for the solution of shear wave equation," International Journal of Nonlinear Sciences and Numerical Simulation, vol. 14, no. 5, pp. 267-273, 2013.

[29] X. F. Chen, J. W. Xiang, B. Li, and Z. He, "A study of multiscale wavelet-based elements for adaptive finite element analysis," Advances in Engineering Software, vol. 41, no. 2, pp. 196-205, 2010.

[30] X. W. Zhang, X. F. Chen, X. Z. Wang, and Z. He, "Multivariable finite elements based on B-spline wavelet on the interval for thin plate static and vibration analysis," Finite Elements in Analysis and Design, vol. 46, no. 5, pp. 416-427, 2010.

[31] Z. B. Yang, X. F. Chen, X. W. Zhang et al., "Free vibration and buckling analysis of plates using B-spline wavelet on the interval Mindlin element," Applied Mathematical Modelling, vol. 37, no. 5, pp. 3449-3466, 2013.

[32] X. C. Wang, The Finite Element Methods, Tsing Hua University Press, Beijing, China, 2002.

[33] X. W. Zhang, X. F. Chen, and Z. J. He, "The construction of multivariable reissner-mindlin plate elements based on B-spline wavelet on the interval," Structural Engineering and Mechanics, vol. 38, no. 6, pp. 733-751, 2011.

[34] Z. B. Yang, X. F. Chen, X. W. Zhang, and Z. He, "Wave propagation modeling in one-dimension structures by the B-spline wavelet on interval finite element," Applied Mechanics and Materials, vol. 105-107, pp. 3-8, 2012.

[35] J. W. Xiang, T. Matsumoto, and Y. X. Wang, "Detect damage in conical shells using curvature mode shape and wavelet finite element method," International Journal of Mechanical Sciences, vol. 66, pp. 83-93, 2013.

[36] X. W. Zhang, X. F. Chen, Z. J. He, and Z. B. Yang, "The analysis of shallow shells based on multivariable wavelet finite element method," Acta Mechanica Solida Sinica, vol. 24, no. 5, pp. 450460, 2011.

[37] P. K. Basu, A. B. Jorge, S. Badri, and J. Lin, "Higher-order modeling of continua by finite-element, boundary-element, Meshless, and wavelet methods," Computers and Mathematics with Applications, vol. 46, no. 1, pp. 15-33, 2003.

[38] S. P. Timoshenko and D. H. Young, Theory of Structures, McGraw-Hill Company, Inc, New York, NY, USA, 1965.

[39] J. C. Goswami, A. K. Chan, and C. K. Chui, "On solving firstkind integral equations using wavelets on a bounded interval," IEEE Transactions on Antennas and Propagation, vol. 43, no. 6, pp. 614-622, 1995.

[40] J. W. Xiang, X. F. Chen, Z. J. He, and H. B. Dong, "The construction of $1 \mathrm{D}$ wavelet finite elements for structural analysis," Computational Mechanics, vol. 40, no. 2, pp. 325-339, 2007. 

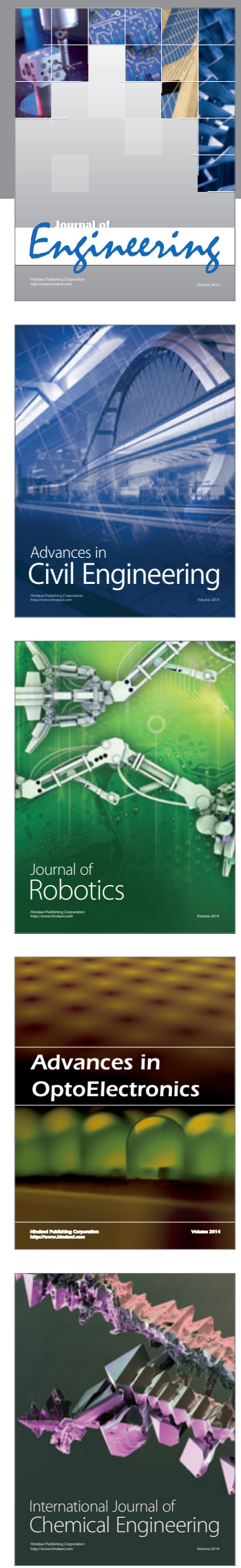

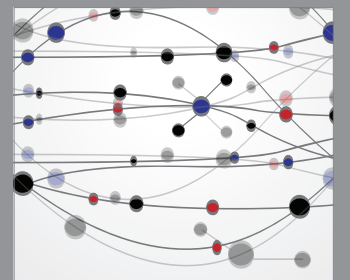

The Scientific World Journal
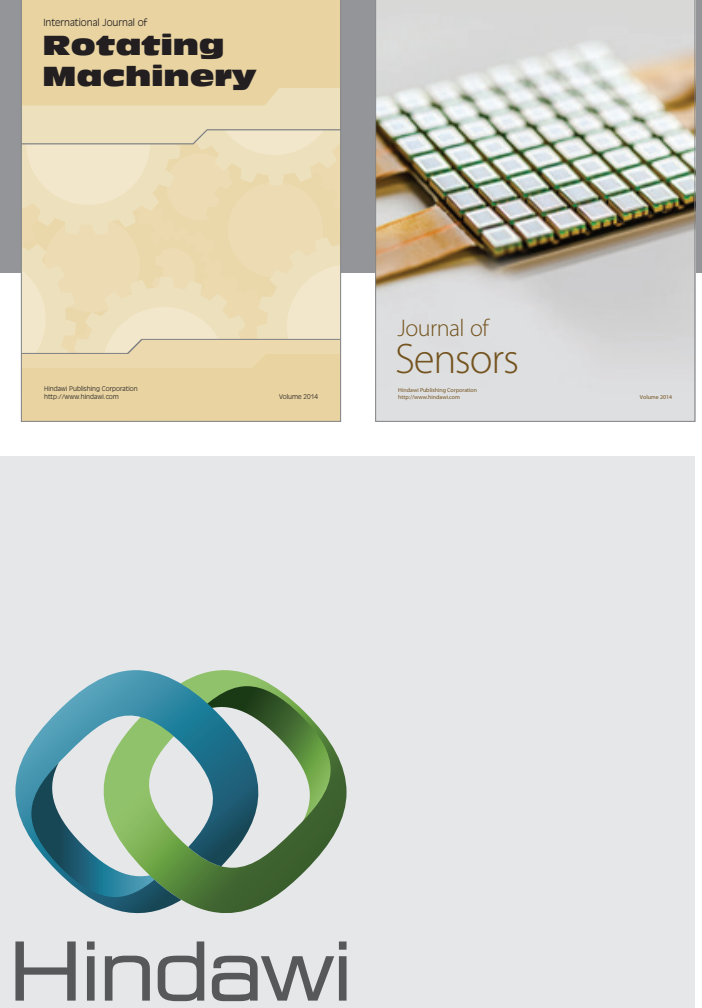

Submit your manuscripts at http://www.hindawi.com
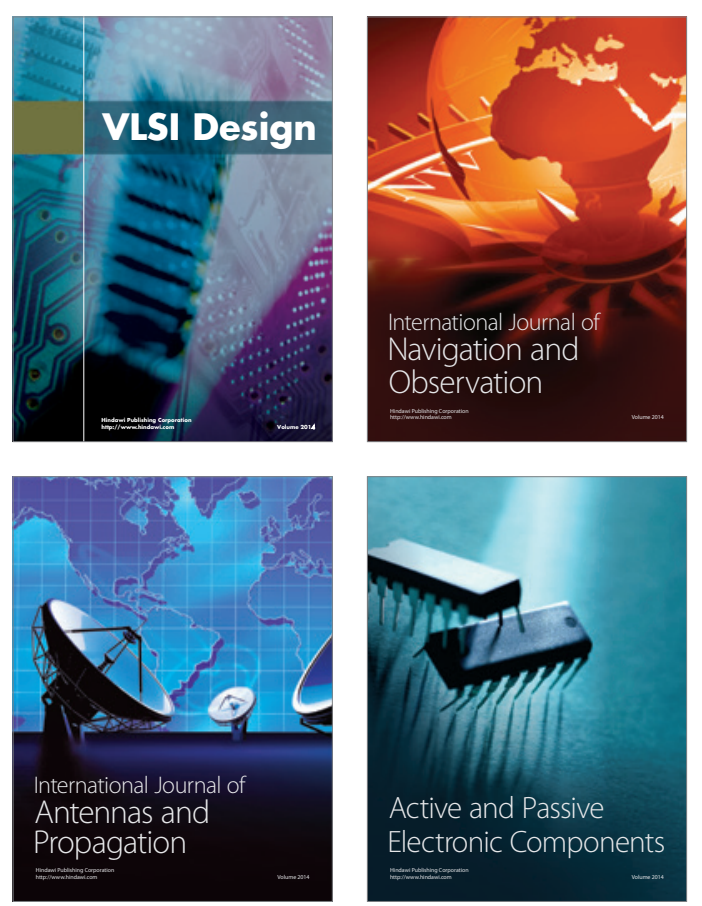
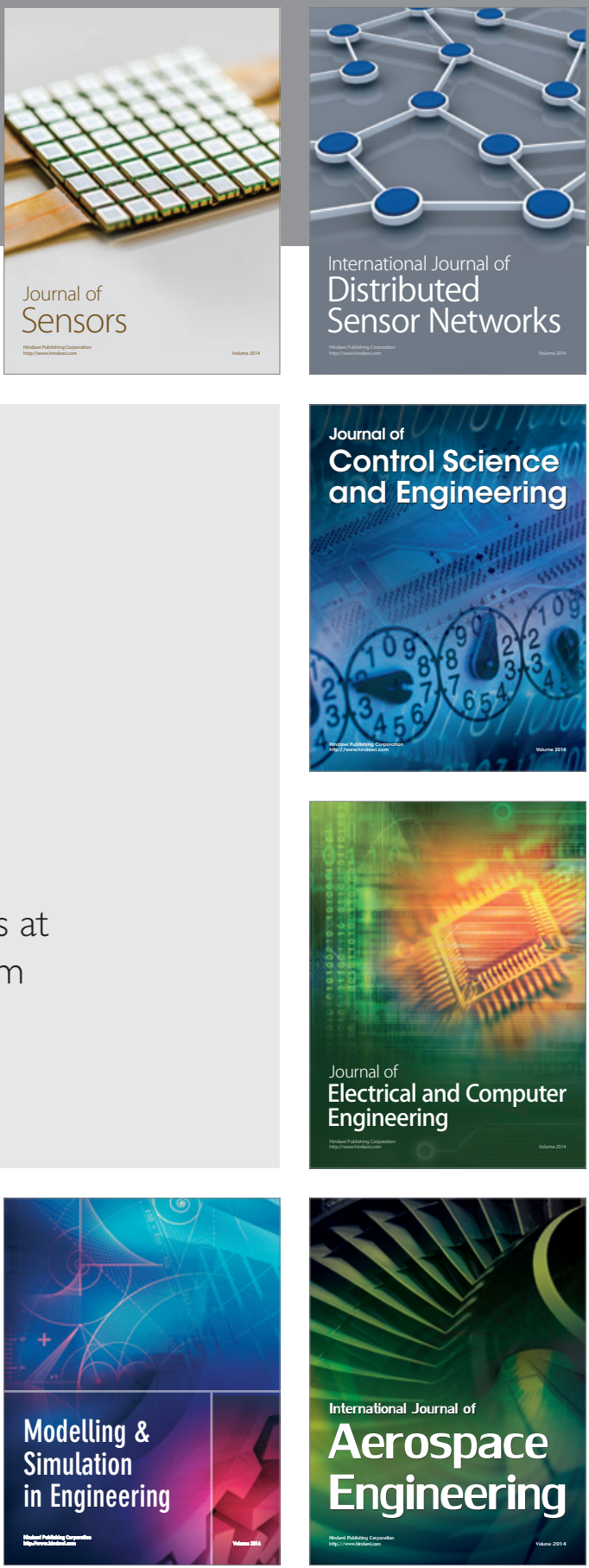

Journal of

Control Science

and Engineering
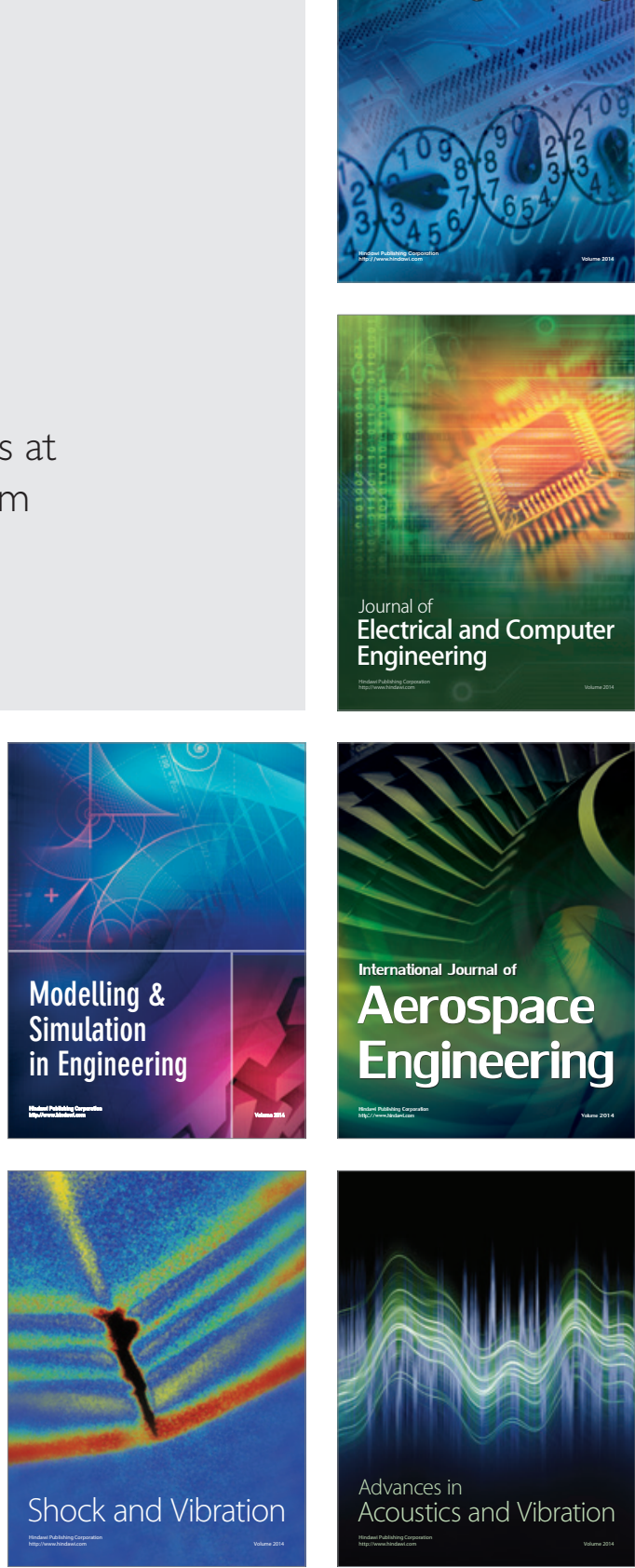